\title{
The magnetic Rayleigh-Taylor instability in solar prominences
}

\author{
Andrew Hillier ${ }^{1}$
}

Received: 1 November 2016/ Accepted: 29 November 2017/Published online: 15 December 2017

(C) The Author(s) 2017. This article is an open access publication

\begin{abstract}
The magnetic Rayleigh-Taylor instability is a fundamental instability of many astrophysical systems, and recent observations are consistent with this instability developing in solar prominences. Prominences are cool, dense clouds of plasma that form in the solar corona that display a wide range of dynamics of a multitude of spatial and temporal scales, and two different phenomena that have been discovered to occur in prominences can be understood as resulting from the Rayleigh-Taylor instability. The first is that of plumes that rise through quiescent prominences from low density bubbles that form below them. The second is that of a prominence eruption that fragments as the material falls back to the solar surface. To identify these events as the magnetic Rayleigh-Taylor instability, a wide range of theoretical work, both numerical and analytical has been performed, though alternative explanations do exist. For both of these sets of observations, determining that they are created by the magnetic Rayleigh-Taylor instability has meant that the linear instability conditions and nonlinear dynamics can be used to make estimates of the magnetic field strength. There are strong connections between these phenomena and those in a number of other astro, space and plasma systems, making these observations very important for our understanding of the role of the RayleighTaylor instability in magnetised systems.
\end{abstract}

Keywords Magnetohydrodynamics (MHD) - Sun: filaments · prominences · Magnetic fields · Instabilities

Andrew Hillier is supported by his STFC Ernest Rutherford Fellowship Grant Number ST/L00397X/2.

Andrew Hillier

a.s.hillier@exeter.ac.uk

1 CEMPS, University of Exeter, Exeter EX4 4QF, UK 


\section{Introduction}

The topic of this review paper is the magnetic Rayleigh-Taylor instability in solar prominences. As such this paper will look at the evidence, both observational and theoretical, that this instability can develop in prominences. Following this, I will look at how the existence of this instability can be used to investigate the conditions in a prominence. Finally, I try to connect the prominence dynamics to other examples of the Rayleigh-Taylor instability found in astrophysical systems.

This review is largely inspired by observations of prominences that have been performed over the last ten years. Using the Solar Optical Telescope (SOT: Tsuneta et al. 2008) onboard the Hinode satellite (Kosugi et al. 2007), observations of quiescent prominences show buoyant plumes, which rise through the prominence material. There is also the observation of a prominence eruption observed by the atmospheric imaging assembly (AIA: Lemen et al. 2012) onboard the Solar Dynamics Observatory (SDO) that fragments as it falls back towards the solar surface in a way that is strongly reminiscent of water splashes and milk crowns.

These observations of the Rayleigh-Taylor instability are, in the authors somewhat biased opinion, the best examples that exist in astrophysics. With the high resolution of the Hinode SOT observations combined with the high temporal resolutions, it provides a wonderful opportunity to understand how the role of the Rayleigh-Taylor instability in astrophysical systems. In this paper, I will look at the observations, analytic theory and the numerical simulations that have been performed to understand the mechanism that forms the plumes and the eruption fragments, and have been used to infer information about prominences through the observations.

This review is laid out in the following way. First, I will provide the necessary background information required to sufficiently understand for the purposes of this review both prominences and the magnetic Rayleigh-Taylor instability. Then I will present information gleaned from observations of prominence dynamics and then present the theoretical work that connects these observations to the magnetic Rayleigh-Taylor instability. At this point, I will also look at the other explanations that have been put forward to explain this phenomenon. I will then present the methods through which the theory of the magnetic Rayleigh-Taylor instability can be used to determine the physical conditions of a prominence. Finally, I will connect between these observations and other astrophysical, space and plasma systems.

\section{Prominences}

The solar corona is made of hot (greater than $1 \mathrm{MK})$ and tenuous $\left(\sim 10^{-15} \mathrm{~g} \mathrm{~cm}^{-3}\right)$ plasma that comprises the outer layer of the solar atmosphere. This is a layer of the atmosphere that is dominated by the magnetic field where the plasma beta, the ratio of gas to magnetic pressure, is less than unity. For more information on the formation of the hot corona see, for example, De Moortel and Browning (2015). 
One of the most striking features in the solar corona are prominences. Prominences are relatively speaking cool and dense condensates that form in the solar corona. There is a long history of prominence observations (e.g. Secchi 1877), mainly during eclipses when the bright light of the solar disk is blocked, allowing prominences to be easily observed at the solar limb. Prominences can be observed clearly in cool spectral lines (e.g. $\mathrm{H} \alpha$ ) highlighting their low temperature $\left(\sim 10^{4} \mathrm{~K}\right)$. This is a temperature that is approximately two orders of magnitude lower than that of the surrounding corona. In tandem with the comparatively low temperature of prominences, the density is comparatively high, being approximately two orders of magnitude greater than that of the surrounding corona. The characteristic dimensions of prominences (filaments) are: length of $6 \times 10^{4}$ to $6 \times 10^{5} \mathrm{~km}$, height of $1.5 \times 10^{4}$ to $10^{5} \mathrm{~km}$ and thickness $5 \times 10^{3}$ to $1.5 \times 10^{4} \mathrm{~km}$ (TandbergHanssen 1995).

In the remainder of this section, and in some of the following sections, I will attempt to provide sufficient information on prominences to put this review in context. For more information on prominences, including areas such as prominence formation that I do not address in any detail, and a deeper look at our understanding of them see, for example, Tandberg-Hanssen (1995), Labrosse et al. (2010), or Mackay et al. (2010).

Prominences can be classified into three categories: quiescent, intermediate and active region (e.g Tandberg-Hanssen 1995), where one key aspect for this classification is the strength of the photospheric magnetic field. Active region prominences are associated with the magnetic neutral lines of the strong magnetic fields that manifest as sunspots and active regions. As the photospheric field becomes weaker further away from active regions, the visible characteristics of the prominence change. Once we reach the weak field strengths of the quiet sun, then we have what are known as quiescent prominences. Figure 1 shows Hinode SOT observations of an active region prominence. A quiescent prominence is shown in Fig. 2.

Prominences do not exist purely at the solar limb, the rotation of the Sun dictates that anything observed on the limb will eventually rotate around and be present on

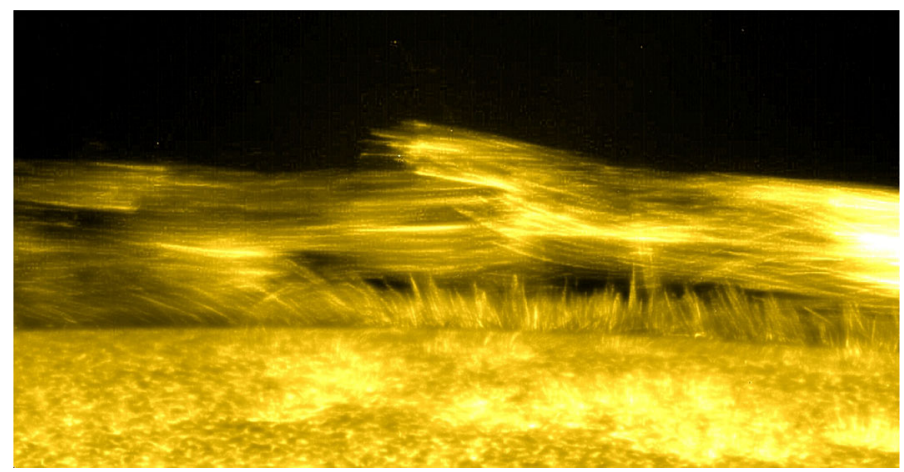

Fig. 1 An active region prominence observed on 8 February 2007 by Hinode SOT, courtesy of J. Okamoto (NAOJ). The dynamics of this prominence are analysed in Okamoto et al. (2016) 


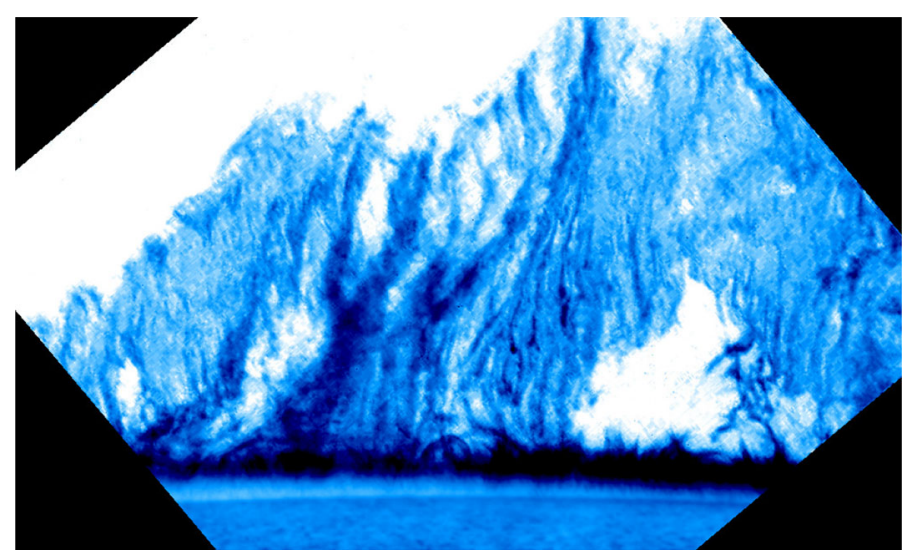

Fig. 2 A quiescent prominence observed by Hinode SOT using the Ca II H broadband filter on 29 September 2008. The image is shown as a negative to highlight the prominence features

the solar disk. While prominences are seen as features in emission in spectral lines at the solar limb, on the solar disk they appear as dark absorption features. These are known as filaments. Using measurements of the photospheric magnetic field, it is known that filaments form in filament channels above the polarity inversion line, the line along which the radial magnetic field changes from positive to negative. As they are fundamentally the same phenomenon, in this review, I will use the two terms interchangeably.

Prominences, as with any other coronal structure, are magnetised and measuring the strength and orientation of the magnetic field is one key part of understanding prominences themselves. The magnetic field, though, is not something that can be directly measured, it has to be inferred from the measurement of what are know as the Stokes parameters which show the linear and circular polarization of the light as a result of the presence of a magnetic field. From these it is possible to infer the strength and direction of the prominence magnetic field. For quiescent prominences generally the magnetic field strengths are between 3 to $30 \mathrm{G}$, though patches of the prominence are often measured to have much larger field strengths (e.g. Leroy 1989; López Ariste et al. 2006; Casini et al. 2009; Schmieder et al. 2013; Orozco Suárez et al. 2014; Levens et al. 2016).

\subsection{Prominence models}

Before delving into the observed dynamics of prominences, it makes sense to spend some time outlining the possible methods by which prominence material is supported against gravity. What we require is a force that can balance gravity.

With a view to discarding it, we will first look at gas pressure to support the prominence. The temperature of a prominence is approximately $10^{4} \mathrm{~K}$. The pressure scale height associated with this temperature is of the order of a few hundred kilometres, which is about two orders of magnitude smaller than the height of observed prominences. This leads to the conclusion that the support of the 
prominence material against gravity is by the magnetic field. However, as the Lorentz force of the magnetic field is an anisotropic force with no component aligned with the magnetic field. This does mean that though the whole prominence cannot be supported by gas pressure, the gas pressure gradient along magnetic field lines is likely to be important for determining the spatial structuring of prominences.

To support prominence material by a magnetic field, we can think of this happening by either magnetic pressure or magnetic tension. The measurements of magnetic fields in prominences and filaments suggest that there exists a field that is predominantly horizontal at the places where the prominence material is observed (e.g. López Ariste et al. 2006). Therefore, we will look at models where the magnetic field would become horizontal in the prominence.

If we are to construct a model where magnetic pressure supports the prominence against gravity, we can take the idea of a horizontal field and set up an atmosphere where the Alfvén speed and the sound speed are constant, which would imply that the plasma $\beta$ is also constant. Taking a magnetohydrostatic equilibrium of an ideal gas under these conditions gives

$$
\frac{\partial}{\partial z}\left(p+\frac{B^{2}}{8 \pi}\right)=\frac{\beta+1}{\beta} \frac{\partial p}{\partial z}=-p \frac{g \mu}{R T}=-\rho g,
$$

where $\mu$ is the mean molecular mass and $R$ is the gas constant. This has the solution

$$
p(z)=p(0) \exp \left(-\frac{z}{H} \frac{\beta}{\beta+1}\right)
$$

where $H$ is the thermal pressure scale height. It is clear that the inclusion of magnetic pressure in the force balance increases the scale height of the prominence by a factor of $(\beta+1) / \beta$, so that if the plasma beta is $\beta=0.01$ the scale height of a prominence supported by the total pressure would be around that of the observed prominences.

As we have seen, we are able to create a magnetohydrostatic solution using total pressure that could provide the necessary support for prominences. However, there are a number of issues with this model. First, the required plasma beta needed to increase the thermal pressure scale height to the height of prominence plasma may be too small to explain a quiescent prominence. Second, the Brunt-Väisälä frequency of this equilibrium is given as (Goedbloed and Poedts 2004)

$$
N_{\mathrm{B}}^{2}=\frac{(\gamma-1) \beta-1}{1+\beta} \frac{g^{2}}{C_{\mathrm{s}}^{2}},
$$

where $\gamma$ is the ratio of specific heats and $C_{\mathrm{s}}$ is the sound speed of the fluid, and is negative (i.e. the system is convectively unstable) when $\beta<(\gamma-1)^{-1}$. Though we are looking at a very specific case of possible pressure support models, it does highlight the potential limitations of purely invoking a total pressure gradient to support prominences. Third, the magnetic field model taken here is very simplistic compared to the actual magnetic field in the solar atmosphere, where field lines generally come out of and then re-enter the solar surface. Therefore, we do not 
expect the main support of prominence material against gravity to come from the total pressure gradient.

This leaves magnetic tension as the key force to support the plasma against gravity. By comparing the two forces, gravity and magnetic tension, it is possible to estimate the necessary curvature of the magnetic field to support a prominence for a given magnetic field strength. Using a magnetohydrostatic balance, we have

$$
\begin{gathered}
\frac{B_{x}}{4 \pi} \frac{\partial B_{z}}{\partial x}=\rho g \\
\rightarrow L \sim \frac{B^{2}}{4 \pi \rho g},
\end{gathered}
$$

where $L$ is the radius of curvature of the magnetic field and gives the necessary curvature to support prominence material against gravity. Taking a field strength of $100 \mathrm{G}$ and the density of $10^{-13} \mathrm{~g} \mathrm{~cm}^{-3}$ would lead to a lengthscale of

$$
L=3 \times 10^{11}\left(\frac{B}{100 \mathrm{G}}\right)^{2}\left(\frac{\rho}{10^{-13} \mathrm{~g} \mathrm{~cm}^{-3}}\right)^{-1} \mathrm{~cm} .
$$

However, for quiescent prominences where the field strength can be significantly lower (i.e. $B$ may only be 3 Gauss), this lengthscale would reduce by about three orders of magnitude, becoming about $3000 \mathrm{~km}$. This has now become a lengthscale that is approximately the same as those observed in quiescent prominence dynamics (e.g. Hillier et al. 2017), i.e. we are now in a regime where the force of gravity has become significant on the observable lengthscales when compared to the magnetic forces. This comes as no surprise due to the highly dynamic nature of quiescent prominences, which display a large amount of structuring and flows that are aligned with the direction of gravity (see the description of prominence dynamics in Sects. 2.2 and 2.3).

Aulanier and Demoulin (1998) developed a model for estimating the prominence magnetic field structure based on extrapolating a linear force-free magnetic field from the magnetic field of the solar photosphere. Using a simple model of the photospheric magnetic field that captures the main characteristics of the magnetic field observed in filament channels (i.e. a polarity inversion line with parasitic polarities) they looked at the position of the dips in the extrapolated magnetic field under the hypothesis that this is where the prominence material will collect. By looking at these, they found a vertical sheet with feet extending into the regions of parasitic polarity. This generally resembles the large-scale structure of a prominence/filament. Aulanier and Démoulin (2003) extended this model to include observed magnetograms finding that for a quiescent prominence their model gave field strengths of $\sim 3 \mathrm{G}$, weak gradients of the magnetic field with height and predominantly inverse polarity with angles of the magnetic field of $\sim 20^{\circ}$ to the prominence axis. Other models based on construction of a force-free magnetic field, including the flux rope insertion model (Su and van Ballegooijen 2012), have also be successful at explaining the large-scale structure of prominences. This highlights 
the general importance of the position of dips in the magnetic field, and with it the role of magnetic tension, in the support of a prominence.

One of the simplest models that has been developed to explain the support of prominence material is the Kippenhahn-Schlüter (KS) prominence model (Kippenhahn and Schlüter 1957). This model is based on the concept of a normal polarity prominence, i.e. the direction of the magnetic field across the polarity inversion line is the same as that of a potential field calculated from the same photospheric magnetic field. One way of imagining this prominence model is that material condenses at the apex of a loop of magnetic field in the solar corona. This condensed mass then pulls down the apex, creating a local dip in the magnetic field where magnetic tension can support the dense material against gravity. The necessary and sufficient stability conditions for this type of prominence are (Anzer 1969)

$$
\begin{aligned}
& {\left[B_{z}\right] \frac{\mathrm{d} B_{x}(x=0)}{\mathrm{d} z}>0,} \\
& B_{x}(x=0) \frac{\mathrm{d}\left[B_{z}\right]}{\mathrm{d} z}>0,
\end{aligned}
$$

where [ ] denotes the jump in the horizontal direction across the filament. The simplest form of this model is invariant in the vertical direction and is stable to both linear and nonlinear Lagrangian perturbations (Aly 2012). As an aside, it is interesting to point out the similarity between the KS prominence model and the those of galactic spurs proposed by Mouschovias (1974).

Kuperus and Raadu (1974) proposed a model based on a concentration of current that gives a tube of magnetic flux that is held down by an overlying strapping field. Magnetohydrostatic models of inverse polarity prominences by Blokland and Keppens (2011a) were investigated for the spectrum of waves and instabilities by Blokland and Keppens (2011b), finding convective continuum instabilities. Hillier and van Ballegooijen (2013) developed a 2.5D model (see Fig. 3) of inverse polarity prominences by adding mass to nonlinear force-free fields. They found that the whole magnetic structure of the prominence dropped as a result of the mass loading

Fig. $32.5 \mathrm{D}$ inverse polarity prominence model from Hillier and van Ballegooijen (2013). Magnetic field lines are shown in black; blue denotes the dense prominence region supported by the magnetic field. Note that the bottom of the domain is set to be the solar photosphere and that $\Lambda$ is the coronal pressure scale height. Reproduced with permission

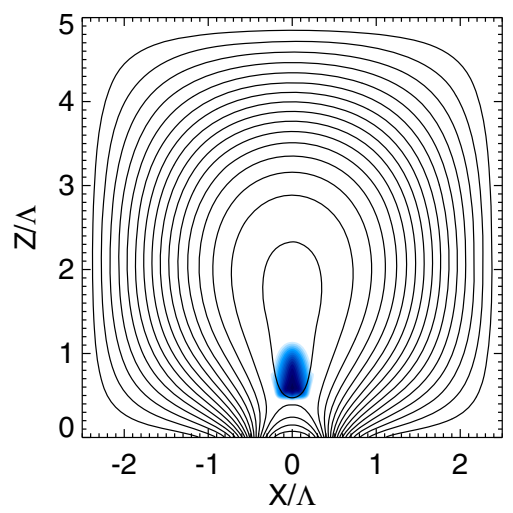


and that the compression of the magnetic field, as well as the stretching of the field, was responsible for the increased tension necessary to support the prominence. Observationally, it has been found that across all types of prominence the inverse polarity prominences are more common than the normal polarity prominences (e.g Bommier and Leroy 1998). Recently, to explain the vertical structuring observed in quiescent prominences, van Ballegooijen and Cranmer (2010) proposed that prominences may be made up of columns of tangled magnetic field which could be formed in a current sheet that develops beneath an inverse polarity flux tube.

As this review is mainly focussed on the dynamics of quiescent prominences, I will not go into detail on the possible mechanisms by which prominence material can appear in the hot corona, though this is a very interesting topic. For example, there has been a great deal of work theoretically on the formation of prominences through radiative condensation in the corona (e.g. Karpen and Antiochos 2008; Luna et al. 2012; Kaneko and Yokoyama 2015; Xia and Keppens 2016a; Kaneko and Yokoyama 2017). Some observations also suggest the formation of prominences through this mechanism (Berger et al. 2012), but other observations are consistent with other mechanisms such as levitation of prominence material by magnetic flux emergence (Okamoto et al. 2010). The review paper by Mackay et al. (2010) covers prominence formation in detail and the interested reader is direct their review and the references it contains.

\subsection{Dynamics of prominences}

The observed dynamics in solar prominences does somewhat depend on the type of prominence being observed. Though thread-like structures dominate all types of prominence (e.g. Lin et al. 2008; Gunár and Mackay 2015 and see Fig. 1), there are many differences. For active region prominences, they are likely to be dominated by field aligned flows and magnetohydrodynamic (MHD) waves (e.g. Okamoto et al. 2007 ) in the horizontal threads, but also can show winding motions (Okamoto et al. 2016). Active region prominences are more eruptive, and as such have shorter lifetimes, but quiescent prominences can remain in the corona for weeks. Quiescent prominences, however, display a much wider range of flow dynamics, especially those orientated in the vertical direction (e.g. Berger et al. 2008), and vertical structuring. Figure 2 gives an example of a quiescent prominence observed by Hinode SOT. In the following part of this section, I will briefly review some key dynamic features of prominences.

Oscillatory dynamics are often observed in prominences, with two distinct classes of oscillations seen. These are known as large amplitude and small amplitude oscillations (Oliver and Ballester 2002). The definition of large amplitude oscillations is that they have a velocity amplitude $>20 \mathrm{~km} \mathrm{~s}^{-1}$. It is often the existence of a large-scale, impulsive driver, as a result of a flare or eruption in the corona disturbing the prominence directly or indirectly that causes these oscillations.

Large-scale wavefronts propagating through the solar corona, observed as, for example, EUV waves and Moreton waves (which are observed as propagating fronts 
in the wings of the $\mathrm{H} \alpha$ line and interpreted as depressions of the chromosphere by a shock propagating in the corona) are one key source of these oscillations. For example, Asai et al. (2012) presented observations of large amplitude oscillations associated with a propagating front observed both as a Moreton wave and an EUV wave, which was interpreted as being a shock wave in the corona, hitting the prominence driving the oscillations. It can be expected that the interaction between a prominence and a shock wave should result in the compression of the material in the prominence. By developing a theoretical model of a shock hitting a prominence, Takahashi et al. (2015) was able to show that the observations were consistent with this interpretation. These oscillations have been used to estimate the strength of the magnetic field in prominences (e.g. Gosain and Foullon 2012).

Small amplitude oscillations are traditionally those that are associated with velocity amplitudes $\leq 10 \mathrm{~km} \mathrm{~s}^{-1}$, though there are observations of small amplitude oscillations that do not match this condition (e.g. Hillier et al. 2013). Initial observations of small amplitude oscillations found periods of a few minutes that led to the hypothesis these were the signature of Alfvén waves driven by photospheric convection (Harvey 1969). In an active region prominence, Okamoto et al. (2007) found standing oscillations of horizontal threads which they interpreted as Alfvén waves. Using Mg II K spectra from the Interface Region Imaging Spectrograph (IRIS) combined with Hinode SOT observations, Okamoto et al. (2015) and Antolin et al. (2015) found that the damped oscillations observed were compatible to the resonant absorption of MHD kink waves. In quiescent prominences, there is a wide range of frequencies of oscillatory motions (Hillier et al. 2013) of the vertical prominence threads that form in quiescent prominences. These results supported the hypothesis that the waves are driven by the convective motions of the photosphere.

It is not only Alfvén and kink waves that have been observed in prominences, there are also possible signatures of compressible waves. Schmieder et al. (2013) found a wave train propagating up through the prominence at a speed of $10 \mathrm{~km} \mathrm{~s}^{-1}$ with a wavelength of approximately $2000 \mathrm{~km}$. Using magnetic field measurements, which inferred the field to be predominantly horizontal of approximately $7.5 \mathrm{G}$, they interpreted the wave train as propagating fast-mode MHD waves. However, to be consistent with this hypothesis, they required the prominence, instead of being a vertical structure to be only at an angle of $15^{\circ}$ to the line-of-sight. Kaneko et al. (2015) presented an alternative interpretation where the phase mixing of continuum Alfvén waves or continuum slow waves could create the illusion of wave propagation. This would be observed as a super-slow propagating wave front, but would not be associated with the propagation of energy. For a review of waves in prominences and their use in determining the physical conditions of the prominence see, for example, Arregui et al. (2012).

Prominences are also full of small-scale flows of materials (Engvold 1981). Chae (2010) presented observations of downflowing blobs called knots using Hinode SOT finding that they were impulsively accelerated to velocities of $\sim 10 \mathrm{~km} \mathrm{~s}^{-1}$. Using the MSDP spectrograph operating in the Meudon solar tower combined with Hinode SOT observations, Schmieder et al. (2010) suggested that due to the similar magnitude to the vertical and line-of-sight component some of the observed flows 
were of material flowing along the magnetic field. There are also observations showing clear vortex motions of the prominence material (Liggett and Zirin 1984). Ahn et al. (2010) used the observed flows in an intermediate prominence to estimate the depth of the dips in the magnetic field finding angles between $13^{\circ}$ and $39^{\circ}$.

With the existence of waves and a wide variety of nonlinear flows, coupled with the large Reynolds and magnetic Reynolds numbers of the system, it is no surprise that prominences can develop turbulence. Using Hinode SOT observations, Leonardis et al. (2012) investigated the correlations between the intensity fluctuations across different positions and times during a prominence observation. They found that the power spectral density of the intensity fluctuations has power law distributions in both space and time, though the exponents of these power laws are inconsistent with those proposed by turbulence theory. Also, the distribution with wavenumber $k$ of the power spectral densities displayed a break in the distribution at scales of the order $10^{3} \mathrm{~km}$. Freed et al. (2016) used a feature-tracking algorithm to investigate the plane-of-sky velocity field of prominences finding that the kinetic energy spectrum was anisotropic. Hillier et al. (2017) used Hinode SOT Dopplergrams to determine the strength of turbulence in a prominence, finding the velocity spectrum was consistent with those expected from MHD turbulence theory, but that the heating rate from this turbulence would be very small.

This review is not concerned with the dynamics laid out above, though they do help set the scene for the physical environment of the prominence. We are interested in the observations of prominence plumes and how they connect to the magnetic Rayleigh-Taylor instability. This review will mainly focus on these flows and why they seem to appear exclusively in quiescent prominences.

\subsection{Prominence plumes}

Prominence plumes are dark, in the cool spectral lines often used to observe prominences, inclusions that rise through the prominence. Figure 4 shows an example of a plume forming from a large void, known as a bubble (see Sect. 2.4) that developed beneath a prominence. This was observed in $\mathrm{Ca}$ II $\mathrm{H}$ using Hinode SOT.

The first observations in the literature of plumes was presented by Stellmacher and Wiehr (1973). In these observations, observed on 12 October 1971, a large cavity about $30^{\prime \prime}$ in size forms beneath the prominence with a plume breaking off from this cavity and then travelling through the prominence before fragmenting. Using $\mathrm{H} \alpha$ broadband images and $\mathrm{Ca}^{+} 8542 \AA$ spectra they were able to determine a few key characteristics of the rising plume they observed. The plume rose through the prominence at approximately $12 \mathrm{~km} \mathrm{~s}^{-1}$. Using the calcium spectra, they could say that the lack of cool emission in the plume was not caused by the material being Dopplershifted out of the bandpass of the broadband filter, that is to say there is a dearth of cool, prominence material in the plumes. They suggested that the cavity/plume formation was created by an instability. However, the prominence was not globally disturbed by this instability, in fact it lasted until the following day. 


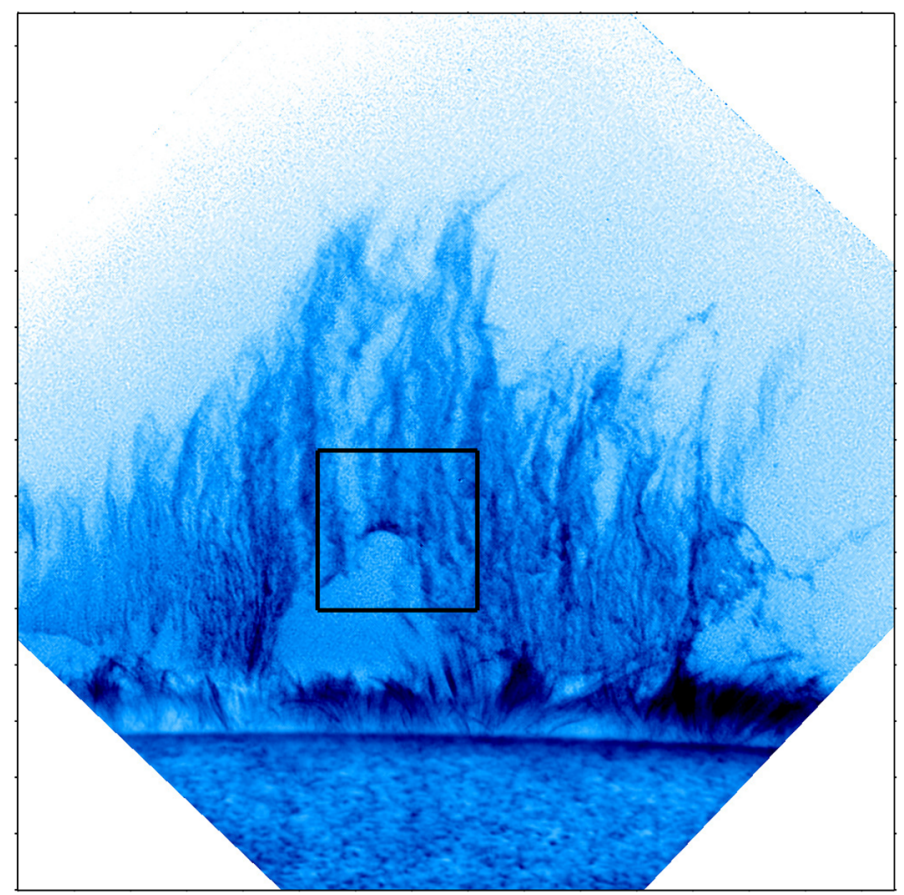

Fig. 4 A plume, marked in the black box, forming as observed in the Ca II H line by Hinode SOT on 3 October 2007. Note the plume's elliptical head and the high intensity emission at the top of the plume. This is a negative image, so dark means high intensity

de Toma et al. (2008) analysed prominence data taken at Mauna Loa Solar Observatory (MLSO) in the $\mathrm{H} \alpha$ line at $656.3 \mathrm{~nm}$ and in $\mathrm{He} \mathrm{I} 1083 \mathrm{~nm}$ on 8 November 2007. They observed a dark bubble, with a core that was bright in $\mathrm{H} \alpha$ rising at a speed of approximately $12 \mathrm{~km} \mathrm{~s}^{-1}$ through the prominence material. They proposed that the bubbles are buoyant, closed magnetic structures that become unstable and rise through the prominence

Those were the observations of quiescent prominences by Hinode SOT that really brought the plumes to the attention of solar researchers. Berger et al. (2008) presented observations of a prominence on 30 November 2006 that underwent the formation of multiple plumes of width $\sim 1000 \mathrm{~km}$ that formed from a bubble that developed at the base of the prominence. The plumes propagated a large way through the prominence body before mixing into the prominence. The authors also noted the brightenings that formed at the plume head and their connection to downflows of prominence material. This led the authors to speculate that the plumes may be adding mass into the prominence which on condensing starts to fall back down. What is telling is that many of the prominence observations with Hinode appear to show the formation of plumes. One implication of this is that the plumes are likely to have always been a common feature of prominences, it is just that it required the seeing free, stable imaging that Hinode SOT provided to reveal this. An example of multiple plumes forming from a bubble is shown in Fig. 5. 

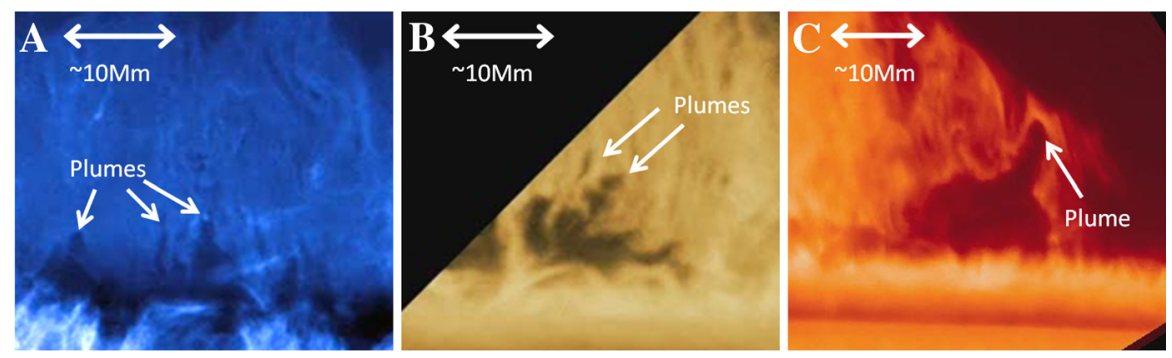

Fig. 5 Different examples of multiple plumes developing from bubbles as observed by Hinode SOT. These were observed on a 30 November 2006, b 8 August 2007 and c 22 June 2010. Figure adapted from Hillier et al. (2012a). Reproduced with permission

Berger et al. (2010) performed the observational analysis of three different prominences to determine some more general characteristics of the plumes. They found that most of the plume lifetime the plume rose through the prominence material at a constant velocity with mean speeds of approximately $16 \mathrm{~km} \mathrm{~s}^{-1}$. After the period of constant rise, the plumes were often found to breakup coincident with a deceleration of the structure. They suggested that the entrainment that occurred as the plume rose through the prominence could be one method to maintain the prominence in spite of the constant downflows of prominence material (c.f. Chae 2010). These authors also presented the hypothesis, first proposed in Ryutova et al. (2010), that the plumes were created by the magnetic Rayleigh-Taylor instability, highlighting that the difference in the direction of the magnetic field between the bubble and the prominence would likely modulate the instability.

Berger et al. (2011) combined Hinode SOT observations with those of the SDO AIA to perform greater analysis of the plume formation. Using $\mathrm{H} \alpha$ Dopplergrams, they also showed that the prominence material is Dopplershifted at the head of the plume as if it is being pushed out the way by the rising plume. This is supported by the ground-based observations of Orozco Suárez et al. (2014) of 2 September 2012 prominence performed using the Tenerife Infrared Polarimeter (TIP-II; Collados et al. 2007) installed at the German Vacuum Tower Telescope at the Observatorio del Teide (Tenerife, Spain). They found that there was a distribution of Doppler velocities across a plume ranging from 0 to $12 \mathrm{~km} \mathrm{~s}^{-1}$. The authors also measured the magnetic field in this prominence finding its strength to be approximately $10 \mathrm{G}$, but no evidence of increased magnetic field strength as a result of compression by the plume was observed.

\subsection{Prominence bubbles}

A large number of the plumes are observed to develop from large bubbles, also called voids and cavities in the literature that form beneath the prominence. It is from the boundary between the prominence and the bubble that the plumes develop and then rise up through the prominence. This has made the nature of the bubbles one of the key questions for understanding how the plumes are formed. Figure 6 


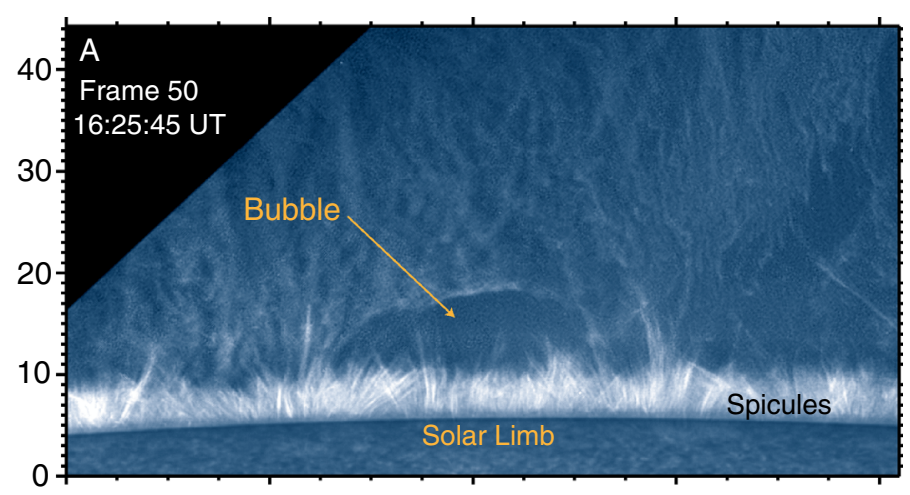

Fig. 6 Bubble observed in a quiescent prominence on 16 August 2007 by Hinode SOT. Adapted from Berger et al. (2017). Reproduced with permission

shows a large bubble that developed beneath a quiescent prominence observed by Hinode SOT. An example of a bubble can also be seen in Fig. 5.

The original paper on the prominence plumes and bubbles (Stellmacher and Wiehr 1973) used $\mathrm{Ca}^{+} 8542 \AA$ spectra to investigate the flows created as the bubble, cavity in their nomenclature, forms. They found blue-shifted Doppler displacements of the bubble border, which they interpreted as prominence material being displaced by the bubble. The use of the calcium spectra also showed that there was a dearth of cool prominence material in the bubble.

Labrosse et al. (2011) investigated the EUV intensity of a prominence with a bubble using Hinode/EIS (Culhane et al. 2007). They found that this bubble appears relatively dark in cool lines where the prominence is in emission and bright compared to the surrounding corona, in coronal lines where the absorption of the line by cool material $\left(10^{4} \mathrm{~K}\right)$ is very effective the bubble appeared bright compared to the prominence. A similar result was found in a bubble observed with $\mathrm{SoHO} /$ SUMER (Berlicki et al. 2011).

Heinzel et al. (2008) compared the intensity of a prominence at $195 \AA$ using both TRACE and Hinode/EIS (though the EIS observations were taken approximately 30 min later) and in X-ray using Hinode/XRT, under the assumption that the relative intensities of the X-ray and EUV lines are the same under coronal conditions to determine the absorption by the prominence. Using this method they were able to estimate the column number density of hydrogen in the prominence to be $\sim 2 \times 10^{-19} \mathrm{~cm}^{-2}$ which is consistent with prominence densities for depths of $\sim 2 \times 10^{3} \mathrm{~km}$. This prominence also had a bubble, and their analysis suggested that the bubble contained cool material with approximately an eighth of the column density of the prominence. I would like to offer a note of caution with this result (other than the possibility of this estimate resulting from foreground or background emission) because in their observations even in regions of the corona away from the prominence differences between the X-ray and EUV emission of $10 \%$ are common, which is a similar departure as measured in the bubble. Using Equations (5) and (6) from the paper, this difference is consistent with optical depths associated with absorption of 
$\tau \sim 0.2$ that are measured in the bubble region. Therefore, in my opinion, though this model is highly effective at determining the opacities and densities in regions with high contrast between the EUV and X-ray emission (i.e. determining the prominence density), the result for the bubble may also be consistent with it containing no cool material at all. Further studies of this type should be performed to see if a consistent absorption feature appears in many bubbles as well as theoretical investigations of this method in the small contrast limit.

Using SDO/AIA Berger et al. (2011) investigated the bubbles that formed beneath two different prominences observed on 22 June 2010 and 2 July 2010 with the aim of determining if there exists any signatures of hot material in the bubbles. They found that there was an excess of emission in the $171 \AA$ channel, which is associated with plasma at around $1 \mathrm{MK}$, for the whole bubble in the 22 June 2010 prominence when the emission was compared to the surrounding corona. Perhaps more interestingly the 2 July 2010 prominence bubble, which was significantly larger, was not filled completely with high emission in the $171 \AA$ channel, but with a transient blob of emission, about $10 \mathrm{Mm}$ in diameter. This blob rose up through the bubble and then burst through the prominence when it reached the prominence bubble boundary. These two events were interpreted as signatures of the existence of hot material in the bubble region. This lead to the theoretical concept that a form of magneto-thermal convection is occurring in prominences, resulting in the observed plumes forming through buoyancy instabilities.

This interpretation is not without its controversy. Dudík et al. (2012) investigated a prominence observed on the northwest limb of the solar disk on 2011 April 20. Using the $193 \AA$ channel of AIA, they found that the emission in this line was approximately the same as the other regions of the corona in the immediate vicinity of the prominence and smaller than the corona further away from the prominence. From this they concluded that there was no evidence that the bubble contained any hot material. However, Berger (2014) re-analysed the same event calculating the differential emission measure, concluding that in this case the bubble was both hotter (though still of the order of $1 \mathrm{MK}$ ) and less dense than the surrounding corona. It should be noted that the large errors in this measurement made strong conclusions difficult.

Gunár et al. (2014) hypothesized that if the dips in the coronal magnetic field existed in front of and behind the bubble and if they were filled with only small amounts of cool material it is likely that the bubble may be clear in $\mathrm{H} \alpha$ but not visible in He II $304 \AA$ due to its greater optical depth. This would mean that any increase in the $171 \AA$ emission could be attributed to the presence of the prominence-coronal transition region (PTCR), which is the region of plasma that forms as the boundary between the hot and cold fluids. In the interpretation of Gunár et al. (2014), the bubble observed on the 22 June 2010 displayed all the signs that the increased emission in the $171 \AA$ passband formed as a result of cool plasma that remained along the line-of-sight either in front of or behind the bubble. When this is the case, it is likely that it would be impossible to distinguish any emission that may be coming from the bubble from that of the foreground and background, that is we can learn nothing about it, showing that this was a possible explanation for the first event in Berger et al. (2011). 
For the second event analysed in Berger et al. (2011), however, where the bubble contained a localised, transient emission in the $171 \AA$ channel, the explanation of Gunár et al. (2014) does not seem to apply. This is simply because the bright blob seen in $171 \AA$ does not have a counterpart in the cooler He II $304 \AA$ band. Therefore, for this event at least this material is not associated with the transition between hot and cold fluid and as such cannot be explained as being PTCR plasma. Only one such event exists in the literature, and an important topic would be to determine the frequency of such events.

The general conclusion that one can give on the debate is that there is some evidence that bubbles contain sufficient plasma emitting at hot enough temperatures to contain their own emission that can be noticeably distinct from that of the corona. However, this is by no means confirmed as a universal feature because in a number of observations it is difficult to distinguish between the emissions of the bubble and the surrounding corona and in some cases foreground or background emission from the PCTR may dominate making it impossible to say anything about what is happening in the bubble itself, and untangling these effects is a difficult task that requires more work. If the bubbles do contain cold material, its density is significantly less than that of the prominence and if any cool material does exist does that imply the existence of dips in the magnetic field inside the bubble (a more complex geometry than in the current models) as otherwise the material would naturally fall to the chromosphere along the magnetic field lines. As can be seen from the variety of results presented in this section, there is still a lot of debate regarding the interpretation of bubble observations but this situation would likely be improved by analysis of more events. One thing that is safe to say that no evidence of densities in the bubble anywhere close to those of the prominence have been found, in fact that some of the densities estimated are of the same order as those of the surrounding corona (Berger 2014).

One area where there is genuine consensus is that the bubble's contain magnetic field (Berger et al. 2011; Dudík et al. 2012). This is to be expected due to the Lorentz force likely dominating gas pressure throughout most of the coronal volume, to form such a large structure is only possible when invoking magnetic field (see arguments for prominence support presented in Sect. 2.1). By modelling a prominence as a linear force free field, that is, by assuming that the prominence is formed of the upward orientated dips in the magnetic field that has a current but zero Lorentz force, and inserting a magnetic bubble underneath it Dudík et al. (2012) were able to show that qualitatively the emergence of magnetic flux beneath a prominence would look like the observed formation of a bubble beneath a prominence. Observationally, there is evidence that flux emergence can occur beneath a prominence (e.g. Chae et al. 2001). Levens et al. (2016) investigated the magnetic field in and around prominence bubbles finding increases in the inferred magnetic field strength in these regions, which the authors interpret as being consistent with the bubble being formed by emerging magnetic flux. This is the first attempt at such measurements and aspects of the results support the ideas behind bubble formation, but cautions has to be taken due to the relatively large space of parameters where solutions to the inversions exist (López Ariste and Casini 2005). 
Based on the low densities expected for the bubbles and their potential magnetic structure, an interesting theoretical idea was presented in Berger et al. (2011). As the bubble is thermally isolated, which prevents thermal relaxation by conduction, and using the heat flux required to heat the solar corona and the typical diameter of the bubbles to estimate the length of a magnetic field line within them, it was possible to show it should take approximately $15 \mathrm{~min}$ before any cool, low-density material they contain heats up to $1 \mathrm{MK}$. Therefore, if any coronal heating process is occurring, we would expect that if the bubble is filled with low-density plasma within a relatively short time it will reach temperatures similar to those of the quiet solar corona.

\subsection{Fragmenting eruption}

The next observation is on a much larger scale than the plumes. However, due to the similarity in the structures created, it seems that the same physical process is at play. The observation concerns the breakup of prominence material as it falls back to the surface of the sun post-eruption as seen on 7 June 2011 and caught attention for two reasons: (1) the dynamics of the breakup of the prominence material as it fell back to the solar surface (Innes et al. 2012; Carlyle et al. 2014) and (2) the bright impacts of material as they hit the solar surface (Gilbert et al. 2013; Reale et al. 2014; Innes et al. 2016). These observations were also used to show how the magnetic field of coronal mass ejections can reconnect with the surrounding coronal magnetic field (van Driel-Gesztelyi et al. 2014).

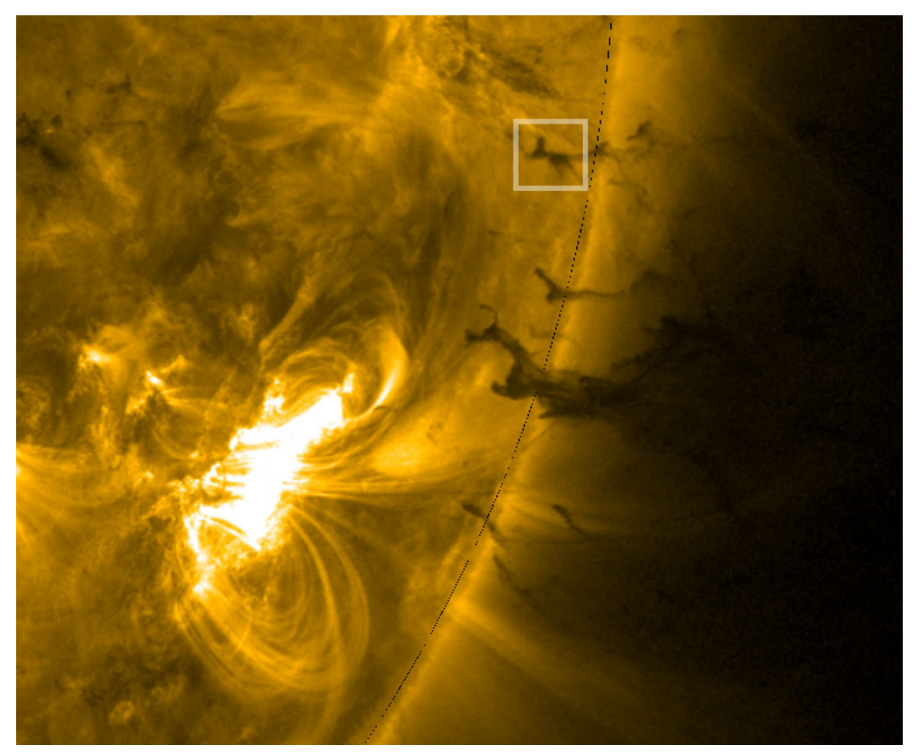

Fig. 7 A fragmenting prominence eruption observed on 7 June 2011. The box shows a fragmenting feature. Image courtesy of Dr. J. Carlyle 
Figure 7 shows the fragmentation of the eruption. As the previously erupted material begins to fragment on its return to the solar surface, a characteristic separation between falling blobs of material of $10^{9} \mathrm{~cm}$ was observed. The blobs of material fell smoothly through the corona where no examples of Kelvin-Helmholtz roll-ups were seen to develop (Innes et al. 2012).

Using polychromatic opacity imaging technique (Williams et al. 2013) to determine the column density of the ejected prominence material as it falls back to the solar surface, Carlyle et al. (2014) found column densities of hydrogen of $2 \times 10^{19} \mathrm{~cm}^{-2}$. This is comparable to the estimated pre-eruption column density of filaments and could be a sign that this filament was particularly massive. Through arguments on the expected volumetric area of the blobs, densities of $2 \times 10^{-14} \mathrm{~g} \mathrm{~cm}^{-3}$ were estimated. The interpretation of why the material breaks up as it falls towards the solar surface is that because of its high density it undergoes the magnetic Rayleigh-Taylor instability. We will look further into this interpretation as part of the rest of the paper.

\section{The magnetic Rayleigh-Taylor instability}

The Rayleigh-Taylor instability is a fundamental instability of gravitationally driven fluid dynamics. The basic concept is that a dense fluid is supported against gravity above a lighter fluid. As this situation has excess gravitational potential energy, the boundary between the two fluids is unstable to perturbations which form rising and falling plumes.

The simplest way of modelling this instability is to look at an incompressible fluid where a region of uniform density fluid is supported above another region of lower density fluid by a pressure gradient against gravity. The growth rate $\sigma$ of the instability under these conditions is given as

$$
\sigma^{2}=g A k,
$$

where $A$ is the Atwood number defined as $A=\left(\rho_{+}-\rho_{-}\right) /\left(\rho_{+}+\rho_{-}\right)$with the + and - symbols denoting the regions above and blow the discontinuity, $g$ is constant gravity and $k$ is the wave number. It is clear to see that if the density is larger above the discontinuity than below then the system is unstable, and if it is smaller then it is stable and produces surface gravity waves. The growth rate of the instability is proportional to $k^{1 / 2}$, therefore the larger the $k$ (and with that the smaller the spatial scale) the faster the instability grows. However, as the vertical component of the eigenfunction of the instability is proportional to $\exp (-k|z|)$, we can already get the feeling that the vertical extent of the linear instability is proportional to $1 / k$. Other arguments based on dynamic considerations produce the same result (e.g. Hillier 2016).

Of interest in many astrophysical systems, and prominences are no exception, is the case where the Rayleigh-Taylor instability is modified by the presence of a horizontal magnetic field. To model the forces of the magnetic field on the fluid, the incompressible ideal MHD equations are used. These are given as 


$$
\begin{gathered}
\rho \frac{D \mathbf{u}}{D t}=-\nabla p+\mathbf{J} \times \mathbf{B}+\rho \mathbf{g}, \\
\frac{\partial \mathbf{B}}{\partial t}=\nabla \times(\mathbf{u} \times \mathbf{B}) .
\end{gathered}
$$

This situation was first investigated for a plasma physics setting by Kruskal and Schwarzschild (1954) where a dense layer is supported above a vacuum. The more general case, where fluid exists both above and below the discontinuity, the growth rate of the instability is given as (Chandrasekhar 1961)

$$
\sigma^{2}=g A k-\frac{(\mathbf{k} \cdot \mathbf{B})^{2}}{2 \pi\left(\rho_{+}+\rho_{-}\right)} .
$$

Here the first term on the right-hand side is exactly the same as the hydrodynamic version of the instability as given in Eq. 9. The second term is that of a surface Alfvén wave and signifies how a magnetic field of strength $B$ in an arbitrary horizontal direction can suppress the growth of the magnetic Rayleigh-Taylor instability through magnetic tension. As the magnetic field has a direction, the influence of this force is anisotropic.

To give a bit more understanding to the development of the magnetic RayleighTaylor instability in a uniform magnetic field, we can think of two fundamental perturbations. These are the interchange mode, where $\mathbf{k} \cdot \mathbf{B}=0$ and the undular mode where $\mathbf{k} \| \mathbf{B}$. The interchange mode, it can be easily seen by looking at Eq. 12, removes the influence of the magnetic field from the instability and reduces to the hydrodynamic instability where the smaller the scale the quicker it grows. The undular mode behaves rather differently. As the magnetic field will work to suppress the instability, there is a play-off between how quickly gravity can drive the formation of a plume and how quickly magnetic tension can suppress it. For the undular mode, simply differentiating with respect to $k$ will reveal the $k$ associated with the fastest growing mode

$$
k=\frac{\pi\left(\rho_{+}-\rho_{-}\right) g}{B^{2}},
$$

where the wave vector is aligned with the direction of the magnetic field. Therefore, the stronger the magnetic field strength the larger the lengthscale at which this most unstable undular mode forms. Due to the fact that the undular mode includes the influence of a suppression term, for the same magnitude of $k$ the interchange mode will always grow faster than the undular mode. The most common mode to be excited in nature is the mixed mode where there is a component of $k$ both parallel and perpendicular to $B$. For more information on the linear stage of this instability see, for example, Hillier (2016).

One well know issue with the magnetic Rayleigh-Taylor instability as laid out in the previous paragraphs is that for certain perturbations (for example, those that have $\mathbf{k} \cdot \mathbf{B}=0$ ) have a growth rate which is unbounded with $k$. Simply put, for certain perturbations, as $k$ goes to infinity so does the growth rate. This issue also exists for the hydrodynamic problem where the problem is often regularized by the 
introduction of a region of finite size over which the density transitions, or viscosity or surface tension are introduced.

One way to regularize the problem through MHD terms, and one which is highly applicable for astrophysical settings, is to allow for the magnetic field to have different directions above and below the contact discontinuity. For uniform field strength both above and below the discontinuity the growth rate of the instability is given as (Ruderman et al. 2014; Hillier 2016)

$$
\sigma^{2}=g A k-\frac{\left(k_{x} B_{x}\right)^{2}+\left(k_{y} B_{y}\right)^{2}}{2 \pi\left(\rho_{+}+\rho_{-}\right)},
$$

assuming that the $x$ direction is the direction of the parallel component of the magnetic field and $y$ is the anti-parallel component. As there are no longer any wave vector that can set the second term on the right-hand side of the equation to be zero, without being zero itself, then magnetic tension will always work to suppress the instability and the higher the wavenumber the greater the suppression. Figure 8 shows how the growth rate of the instability is changed by the inclusion of magnetic shear.

Again, to calculate the most unstable mode, we have to look at the perturbation that maximises the gravitational term while minimising the influence of the suppressive terms from the magnetic field. This in itself is a relatively simple process where first we must ascertain which is smaller $B_{x}^{2}$ or $B_{y}^{2}$. Let us say that $B_{y}^{2}$ is the smaller, then to minimise the second term it is necessary to set $k_{x}$ to be zero. Therefore, the $k_{y}$ that satisfies the following equation:

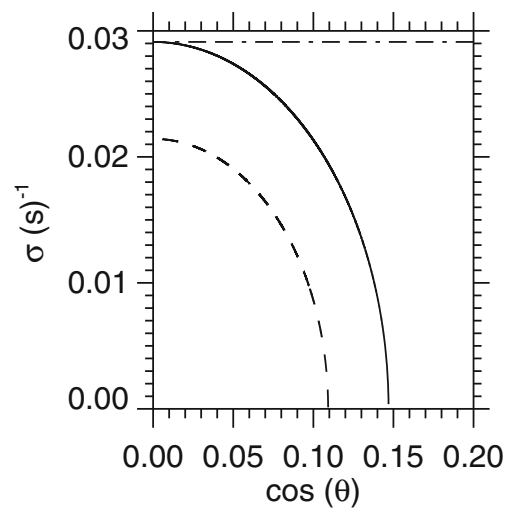

Fig. 8 Growth rate of the uniform field (in the $x$ direction) magnetic Rayleigh-Taylor instability (solid line), the sheared field case (dashed line) for the same field strength and the hydrodynamic case (dotdashed line). In the sheared field case $B_{x}=5 \mathrm{G}$ and $B_{y}=0.5 \mathrm{G}$. Growth rates are calculated using prominence and coronal densities, a wavelength of $2000 \mathrm{~km}$ and $\theta$ giving the angle between the wave vector and the $x$ direction. For the uniform field case, only modes close to the interchange mode grow. The inclusion of the sheared field, in this case only a small amount of shear, noticeably reduces the growth rate 


$$
g A-\frac{2 k_{y} B_{y}^{2}}{2 \pi\left(\rho_{+}+\rho_{-}\right)}=0,
$$

is the $k_{y}$ that gives the fastest growing mode in this system (Hillier 2016). This results in the same calculation as for the fastest going undular mode for a uniform magnetic field of strength $\left|B_{y}\right|$.

Stone and Gardiner (2007a) performed simulations of the magnetic RayleighTaylor instability for a range of shear angles between the upper and lower magnetic field. When the magnetic field is aligned, i.e. zero shear, then long thin structures aligned with the field direction formed. However, the inclusion of shear in the magnetic field results in more isotropic structures forming. Hillier (2016) suggested that this structuring could be formed by the superposition of two plane waves of the same magnitude of $k$ and of growth rate $\sigma$.

Liberatore and Bouquet (2008) investigated the role of stratification and compressibility on the magnetic Rayleigh-Taylor instability in an isothermal plasma, finding that stratification weakens the instability but that compression provided a destabilising effect. Liberatore et al. (2009) compared the theoretical predictions with simulations results finding good agreement.

Ruderman (2017) extended the model of Ruderman et al. (2014) to include compressible effects in the short wavelength limit (i.e. the wavelength is very much smaller than the total pressure scale height-see Eq. 2). They found that compressibility does not change the critical wave vector for the instability that small angles between the magnetic fields in the two regions result in the growth rate matching closely that of the incompressible instability and reducing the plasma $\beta$ only slightly reduces the growth rate. A very interesting result from this work is that adding compressibility breaks the symmetry of the system resulting in the instability being likely to grow perpendicular to the magnetic field in the high density region unlike in the incompressible case.

All the dispersion relations presented until now have looked at a static system, but it is relatively simple to include the influence of a shear flow to the incompressible sheared field dispersion relation. This becomes

$$
\begin{aligned}
\sigma= & i\left[\alpha_{+}\left(\mathbf{k} \cdot \mathbf{U}_{+}\right)+\alpha_{-}\left(\mathbf{k} \cdot \mathbf{U}_{-}\right)\right] \\
& \pm\left[g k A+\alpha_{+} \alpha_{-}(k \cdot \Delta U)^{2}-\frac{k_{x}^{2} B_{x}^{2}+k_{y}^{2} B_{y}^{2}}{2 \pi\left(\rho_{+}+\rho_{-}\right)}\right]^{1 / 2},
\end{aligned}
$$

with $\alpha_{ \pm}=\rho_{ \pm} /\left(\rho_{+}+\rho_{-}\right), U_{ \pm}$being the value of the flow above or below the discontinuity and $\Delta U$ the difference between the two velocity fields. The growth rate shown here can be simply understood as the combined magnetic RayleighTaylor-Kelvin-Helmholtz instability The first term, which is imaginary, just describes the advection of the perturbation by the shear flow. The second term describes the instability, showing that both gravity and shear flow are destabilising terms and magnetic tension a suppression term. The gravity term is maximised by large density differences, but the shear flow term is minimised by these as it scales with $\rho_{\min } / \rho_{\max }$ in the large Atwood number limit where $\rho_{\max }$ is the largest of the 
two densities and $\rho_{\min }$ is the smallest. One interesting result from this equation is that we can have a perturbation that is both Rayleigh-Taylor and Kelvin-Helmholtz stable, but unstable to the combined Rayleigh-Taylor-Kelvin-Helmholtz instability. When they combine, this implies that the scale of the most unstable mode is pushed to smaller scales.

One important consideration is that this growth rate has only been derived for incompressible flows. Once a flow becomes compressible, i.e. if either the slowmode or fast-mode Mach number, or both, of the shear flows are non negligible, then work has to be done to compress the fluid instead of driving the instability and results in a stabilising effect (Miura and Pritchett 1982).

The nonlinear evolution of the Rayleigh-Taylor instability has two key aspects: the formation of the Kelvin-Helmholtz instability and the self-similar evolution of the mixing layer. The Kelvin-Helmholtz instability develops as a result of the shear flows that develop between the rising an falling plumes. Its development is strongly dependent on the density contrast between the two fluids, with larger differences suppressing the development (Daly 1967). The thickness of the mixing layer $(h)$ as a result of the nonlinear development of the instability evolves self-similarly as (Youngs 1989)

$$
h \propto \alpha A g t^{2}
$$

where $\alpha$ is a dimensionless constant measured from hydrodynamic experiments to the between 0.03 to 0.07 (e.g. Dimonte and Schneider 2000). Stone and Gardiner (2007b) performed numerical simulations of the magnetic Rayleigh-Taylor instability finding that the thickness of the mixing layer follows the same scaling law, but the value of $\alpha$ depended of the strength of the magnetic field, with stronger fields having larger values of $\alpha$. This was hypothesized to be as a result of the stronger magnetic field suppressing the development of the Kelvin-Helmholtz instability. It has been discovered that increasing the field strength even more resulted in a reduction in the $\alpha$ value (Carlyle and Hillier 2017). It should also be noted that increased magnetic tension can also work as a nonlinear saturation mechanism in the magnetic Rayleigh-Taylor instability (e.g. Hillier 2016). The 2.5D simulations of the magnetic Rayleigh-Taylor instability of Khomenko et al. (2014) show that the closer to pure interchange modes that are developing, the more chaotic the flows become in the nonlinear regime.

This section has tried to provide sufficient information about this instability to be able to have a sufficient understanding of its nature to aid in the smooth application to solar phenomena. However, there are huge swathes of information that could also be presented. For the interested reader, there are many textbooks with a more complete treatment of the instabilities than this review can provide, for example, Goedbloed and Poedts (2004) and Goedbloed et al. (2010). Also, with particular focus on the Parker modes and their relation to flux emergence in the solar atmosphere then Chapter 3 of Tajima and Shibata (2002) is very informative. 


\section{Theoretical interpretation of the observations}

There have been a small number of interpretations for the formation of the plumes. The Rayleigh-Taylor instability is the interpretation that has been very successful in explaining the plume observations, and as a result has become the accepted theoretical interpretation. However, the question as to whether Kelvin-Helmholtz modes may dominate the formation in some cases (Ryutova et al. 2010), other buoyancy instabilities (Hurlburt and Berger 2012) or whether magnetic reconnection (e.g. Dudík et al. 2012) can form the plumes has also been raised, so I will also present those ideas and try to present the arguments both for and against them.

\subsection{The Rayleigh-Taylor instability}

The first paper that discusses the possibility of the magnetic Rayleigh-Taylor instability developing in prominences is that of Dolginov and Ostryakov (1980). This paper, probably because it was published long before the Hinode SOT observations of plumes, does not look at plume formation but at the possibility for this instability to drive the global disruption of the prominence or the formation of streams of cool material from the prominence. The model employed was a uniform horizontal field, but including the influence of compression. The key results from this paper, as relevant to the formation of prominence plumes, are that

1. This instability requires that the density above the discontinuity is greater than the density below (that is the basic condition for the Rayleigh-Taylor instability is satisfied). This is exactly the condition under which the plumes are observed to form.

2. For small magnetic field strengths, consistent with those of quiescent prominences, the instability will form on small scales with wavelengths of hundreds or thousands of kilometres.

With this, we can see that the theoretical basis for understanding the plume formation in terms of the magnetic Rayleigh-Taylor instability was established well before they became a popular target for observational studies.

Using the Rayleigh-Taylor instability, Cao et al. (1983) tried to explain the disappearance of prominences. Invoking arguments similar to those associated with the bad curvature of magnetic fields leading to ballooning modes, they hypothesized that this may be able to drive the interchange instability in a KS prominence. This concept is similar to the motivation of Anzer (1969) in studying the stability conditions for the KS prominence model.

With the advent of the Hinode observations of plumes, it was not long before the Rayleigh-Taylor interpretation was applied to the observations. Ryutova et al. (2010) was the first to realise that this instability could be key. They listed a set of key criteria that would they felt were requirements that the observed plumes had to conform to for the Rayleigh-Taylor hypothesis to be valid

1. A multi-mode front.

2. Self-similarity of plumes in one family. 
3. Similarity of wavelength/height ratio in different families (i.e., larger wavelength, higher plumes).

4. Suppression of the regular oscillations of filaments comprising the prominence

Ryutova et al. (2010) presented a set of observations of plumes that was consistent with these predictions, and as such concluded that the Rayleigh-Taylor instability was responsible for their creation. Note that in Fig. 8, prominence parameters are used and timescales for the instability of $\sim 100 \mathrm{~s}$ are obtained.

This interpretation was further developed by Berger et al. (2010). They pointed out that even though a magnetic field may suppress the instability, this suppression can only happen along the direction of the magnetic field. Therefore, in the simplest interpretation of the plumes using the magnetic Rayleigh-Taylor instability with a purely uniform field then the interchange mode will always be unstable. To explain the plumes always forming at consistent scales, they suggested that the magnetic field might be sheared between the prominence and the bubble. They also noted that there is a lack of downflows to accompany the upflowing plumes, somewhat counter to the most common view of the Rayleigh-Taylor instability. However, in the limit where the density difference is large, as is expected for the bubble-prominence interface, then the instability enters the so called bubble limit and where the lower fluid penetrates the above fluid as large, distinct bubble-like plumes with the upper fluid coming down as thin spikes of dense material (Daly 1967).

Terradas et al. (2012) investigated that a model of a dense slab of plasma is surrounded above and below by a low density atmosphere. By applying this model to prominence threads, they found that the strength of the magnetic field in active region prominences would suppress this instability for a wide range of perturbations. However, quiescent prominences were more unstable. This may give a reason as to why the plumes are regularly observed in prominences, but there are no such observations in active region prominences.

Though there is as yet no linear analysis that takes into account the complex magnetic structure representative of that of a prominence and a bubble (e.g. Dudík et al. 2012). However, we can perform a thought experiment to gain some understanding about how the instability developing at the top of a magnetic bubble might alter its characteristics. Taking a simple model of a low-density hemisphere (modelling the observed bubble) embedded in a dense surroundings with a constant gravity $\mathbf{g}$, the component of gravity perpendicular to the surface of the hemisphere would be given by $g \cos \theta$. Therefore, we would expect that the peak in the component of the gravity perpendicular to the boundary would be where $\theta=0$, i.e. at the top of the bubble.

Our thought experiment about the nature of the instability in these conditions can lead to three hypotheses. First, that the instability will grow where the gravity is strongest, so plumes can be expected to form near the top of the bubbles instead of at their sides. This is consistent with the observed plumes (see Fig. 5). Second, the component of gravity parallel to the bubble at an angle $\theta$ to the vertical is given by $g \sin \theta$. As $\theta$ increases, the timescale for mass to fall along the edge of the bubble decreases as the timescale for the Rayleigh-Taylor instability increases (as the perpendicular component of gravity is reduced), meaning that for large $\theta$ material is 
more likely to fall along the edge of the bubble to lose gravitational energy than become unstable to the Rayleigh-Taylor instability. We can go a bit further and say that this might imply that if the plume wavelength is small then multiple plumes can form at the top of the bubble (see Fig. 5), though for plumes that are a significant fraction of the bubble radius are going to form as single isolated plumes (see Fig. 4). Third, assuming the bubble to be magnetic, then the mean gravity projected onto a field line that crosses the top of the bubble is given by

$$
g_{\text {mean }}=g \frac{\int_{-\pi / 2}^{\pi / 2} \cos \theta \mathrm{d} \theta}{\int_{-\pi / 2}^{\pi / 2} \mathrm{~d} \theta},
$$

where this is likely to result in an effective gravity driving the instability to be approximately $2 / \pi$ of the surface solar gravity.

\subsubsection{Local simulations of prominence plumes}

The work of Hillier et al. (2011, 2012a) represented the first attempt to numerically model this instability in a prominence. These studies used the KS prominence model, performing local simulations of a prominence. As the KS model used is stable to Lagrangian perturbations (e.g. Aly 2012), just giving a velocity perturbation to this model would not reproduce the desired dynamics. However, the prominence plumes are formed from the boundary between the prominence and a bubble, removing mass from a region of a few pressure scale heights in height and width at the centre of the prominence to create a buoyant bubble worked to create the necessary initial conditions. By removing the density, but keeping the pressure the same, the horizontal force balance of the KS model was maintained, but the vertical force balance was broken meaning that initially the bubble was buoyant. The boundary conditions applied allowed the magnetic field freedom to move, but be kept angled upward.

In the simulations, a multi-mode perturbation was excited using a velocity perturbation centred on the bubble-prominence boundary. First the buoyant bubble rose, but then the perturbations to the boundary grew creating plumes of low density material that rose through the prominence. Due to the nature of the instability, small scales grew quickest, but then larger scales of the instability developed. This process was determined to be a combination of larger linear modes growing, in conjunction with an inverse cascade process driven by vortex interaction. Due to the strong magnetic field, modes close to the interchange mode dominated the evolution. Figure 9 shows how the instability developed in one of the simulations. As well as the simulations without a guide field, Hillier et al. (2012a) also performed simulations with a magnetic field angled at $28^{\circ}$ to the axis of the prominence (i.e. a change in angle of the horizontal magnetic field of $62^{\circ}$ ) and an increase in the strength of the magnetic field at the centre of the prominence of a factor of 3 . The instability also grew in these simulations, and as with the other simulations, the simulated plume structure was dominated by modes that created structure 

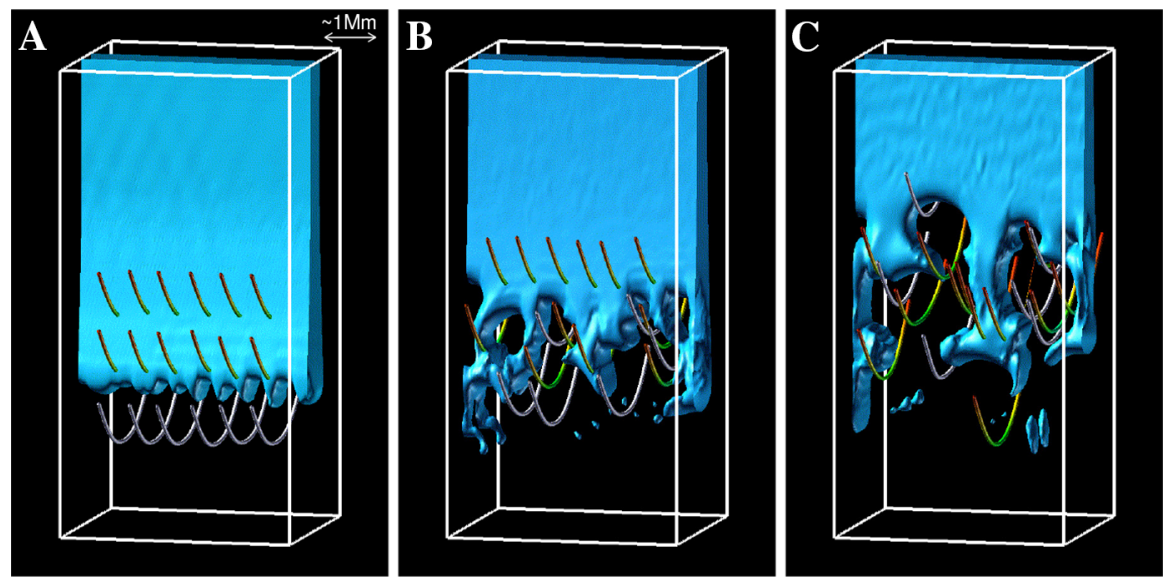

Fig. 9 Evolution of plumes driven by the magnetic Rayleigh-Taylor instability in the KS prominence model presented in Hillier et al. (2012a). Reproduced with permission

perpendicular to the magnetic field. This does imply that the viewing angle to the magnetic field may influence the plume structure observed.

The simulated plumes were qualitatively and quantitatively similar to the observed plumes. The general shape of round-headed plumes with thin falling spikes was the same between the two systems. The plumes simulated reached constant velocities up $\sim 7.5 \mathrm{~km} \mathrm{~s}^{-1}$, which is a few $\mathrm{km} \mathrm{s}^{-1}$ smaller than the slowest of the observed plumes. The simulated velocities depended on the density difference between the prominence and bubble used, suggesting that more realistic density differences may result in more accurate plume velocities. Figure 10 gives a qualitative comparison between the observed plumes and a 2D slice of the simulated plumes from Hillier et al. (2012a).

The general plume morphology is that the plume head is like a low-density cylinder aligned with the magnetic field. Therefore, it can be expected that the plumes are dark in the cool spectral lines because they push the prominence material out the way, removing any emitting material along that line-of-sight.

One unexpected result of these simulations was presented in Hillier et al. (2012b). The formation of rising and falling plumes gave rise to shear flows that resulted in the formation of current sheets. In some of the simulations, these current sheets would reconnect. The reconnection would allow material to fall along the magnetic field and then it would shock. This would result in a dense, supersonic downflow forming that pulled the magnetic field down with it as it fell. This reconnection is similar to that investigated by Petrie \& Low (2005), where they hypothesized the formation of downflowing material, shocks and counterstreaming flows. This model has some resemblance to the model for prominence knots presented by Chae (2010). Observations presented in Hillier et al. (2012b) of one downfalling blob seemed to show the correlation between acceleration and increased intensity that would be expected from shock formation. 

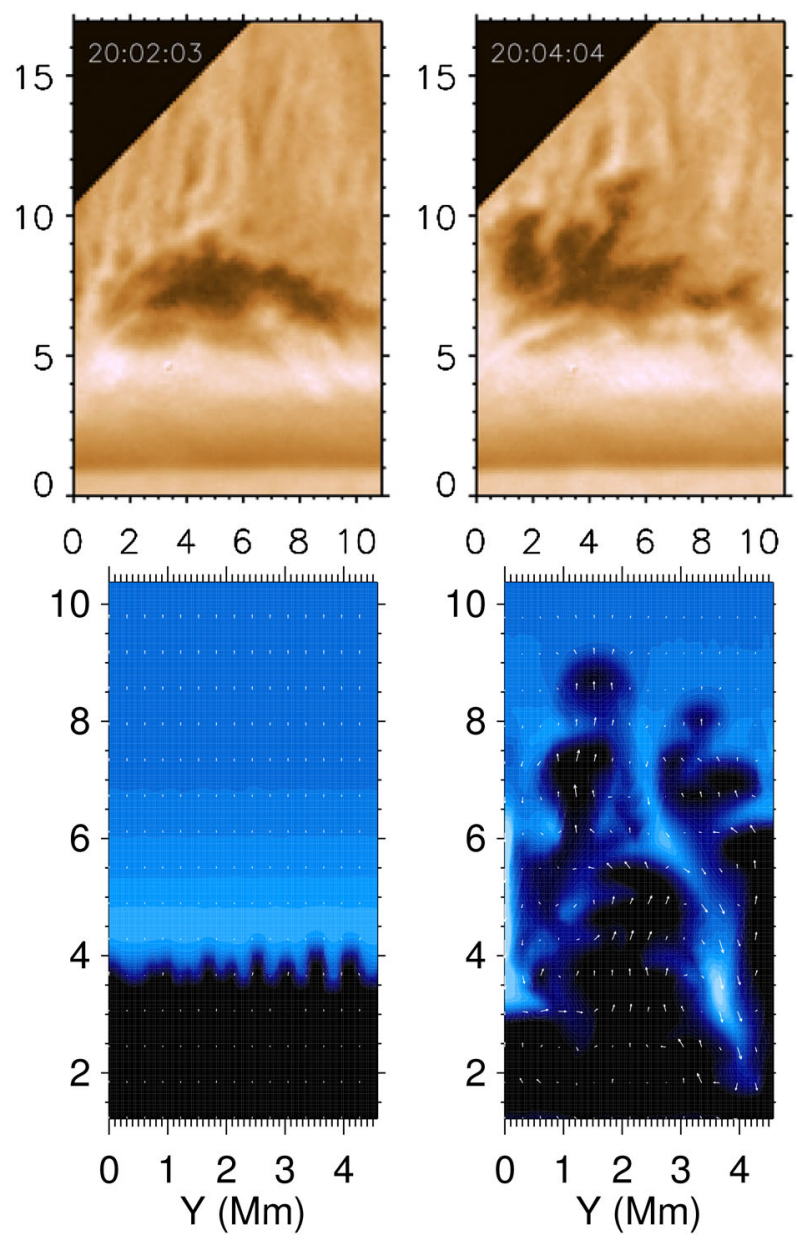

Fig. 10 Comparison between plumes observed on 8 August 2007 top) and simulated plumes. In the simulations, presented in Hillier et al. (2012a), the colour contour shows the density and the arrows give the velocity field. Reproduced with permission

\subsubsection{Simulating this instability in a global prominence model}

The previous subsection looked at the development of the instability caused by a density inversion inside a local model, now we will go beyond this to look at the development of the instability in global models. Here we are defining global by models in which the height and width of the prominence, though not necessarily the length, are contained within the calculation domain.

Keppens et al. (2015) used a magnetically levitated prominence model to investigate the development of Rayleigh-Taylor plumes. They investigated two field strengths, $\sim 8$ and $\sim 20 \mathrm{G}$ (equivalent to plasma $\beta$ values down to $\sim 0.01$ ), finding Rayleigh-Taylor activity in both. The instability resulted in rising and 
falling plumes of a few thousand kilometres in size. The plume structure formed in these simulations is shown in Fig. 11.

Xia and Keppens (2016b) numerically investigated the development of plumes in a model similar to the slab model analytically investigated by Terradas et al. (2012) and numerically by Keppens et al. (2015) but in this case separated into two slabs. Due to the connection of the magnetic field between these two slabs, the evolution of the two was very similar. The instability resulted in the formation of vertical structuring in the prominence, which when viewed from above could be seen as the horizontal structuring. They conjectured that this could be provide an explanation to connect between the observed vertical threads in prominences and horizontal threads in filaments.

Terradas et al. (2015), using a 3D extension of the 2D model of a normal polarity prominence presented in Terradas et al. (2013), investigate the formation of vertical structuring in a 3D prominence model as a result of the magnetic Rayleigh-Taylor instability. By loading mass as to form a low-lying prominence (height $10^{4} \mathrm{~km}$ ) onto the magnetic field and then letting it relax to find a new equilibrium, they found that it went Rayleigh-Taylor unstable. In this case, the size of the instability and the timescale of its growth was determined by the density gradients and the shear in the magnetic field, both compared to the reference potential field and the change in angle of the field with height. They found that for large shear, which had a higher field strength and a faster change in direction of the field with height, that the growth rate of the instability was reduced and for some cases in their model suppressed. One key result from this work was to show that the instability could still grow when

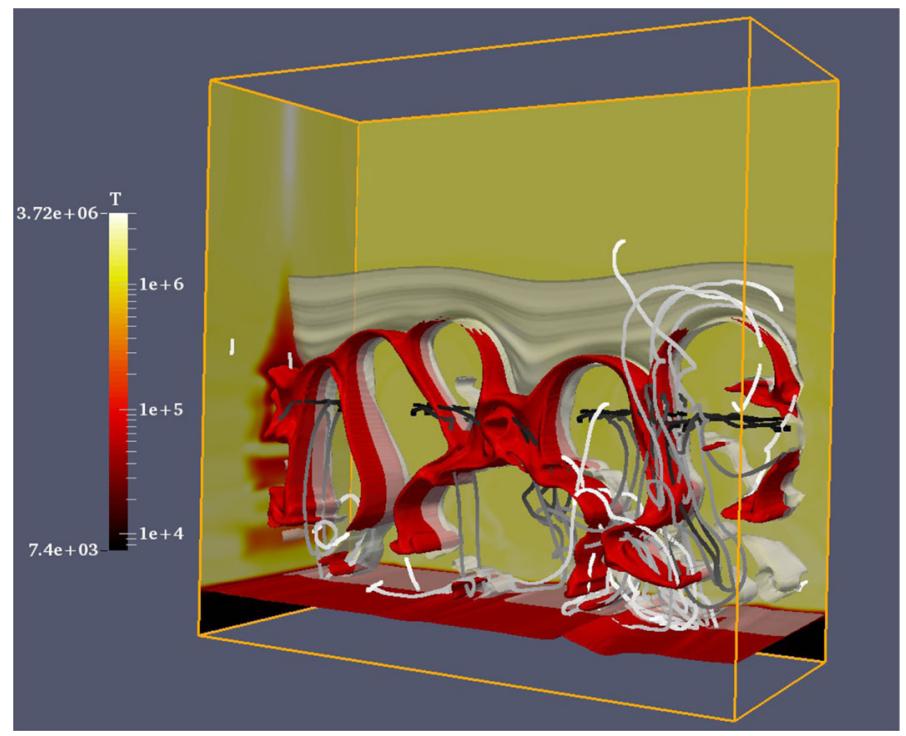

Fig. 11 Plume formation via the magnetic Rayleigh-Taylor instability in a magnetically supported prominence model. Reproduced with permission from Figure 3 of Keppens et al. (2015) @AAS 
the effects of line-tying of the magnetic field, which results from the magnetic field being rooted in the dense solar photosphere, are taken into account.

To think about how different parameters might change the stability of the model, especially in regimes closer to those of a quiescent prominence it is interesting to imagine what would happen if quiescent prominence heights (approximately five times those used) and lengthscales. This would result in the fieldlines in the calculation becoming five times longer and this will ultimately decrease the tension suppression of the instability by a factor of 25 (see second term on RHS of Eq. 12) increasing the growth rate of the instability. Also, using a smaller density transition (about $2 \times 10^{3} \mathrm{~km}$ in their model to make it easy to solve numerically) this would allow smaller scales across the field to become unstable, because of the reduction of suppression from density gradients and a reduction in the vertical change in field direction at that lengthscale, ultimately increasing the growth rate (see first term on RHS of Eq. 12). It is likely that as long a small enough scales across the field are allowed to grow the system will be able to become unstable (Hillier 2016), though this can only be confirmed by performing these calculations.

Terradas et al. (2016) investigated the development of the Rayleigh-Taylor instability in a Titov-Démoulin flux tube (Titov and Démoulin 1999), i.e a standard model for active region filaments. Prominence densities of 30 times that of the corona are used to stop the prominence collapsing. They found that the tightly wound tube resulted in quick saturation of the Rayleigh-Taylor instability giving some suggestion as to why plume dynamics are not observed in active region prominences. As with the models of Terradas et al. (2015), to connect these to quiescent prominence changes in height and the thickness of density transition (along with larger density contrasts) are possible changes to the model, and intuitively one can expect that these changes would increase the growth of any instability in the model (but calculations should be performed to confirm this).

Though not a simulation of the Rayleigh-Taylor instability in a prominence but of prominence formation, the results of Xia and Keppens (2016a) can inform somewhat on the structure of gravitationally driven flows in a quiescent prominence. They presented simulations where an inverse polarity prominence was formed through condensation. Initially, the magnetic field of the simulation was a linear force-free sheared arcade, and through the imposition of shearing and converging flows on the lower boundary during the initial stages of the calculation a flux rope is formed beneath this arcade. A chromosphere was then added, as well as a coronal heating term, and a thermal equilibrium was developed. As with the prominence formation models described in Sect. 2.1, heating at low heights was then applied to the model causing evaporation of the dense plasma at the base of the calculation domain, creating over-dense regions in the corona and then radiative condensation develops creating a prominence. The plasma $\beta$ of the model was approximately 0.2 in the prominence threads. As the material that forms the prominence condenses, it collects in the local dips in the fieldlines. Due to the excess of gravitational potential energy, the fieldlines were dragged down, creating vertical structures in the prominence. The dynamics of this is similar to those presented in Terradas et al. (2015), which were driven by the magnetic Rayleigh-Taylor instability. 
It would be an interesting and necessary development in the investigation of the Rayleigh-Taylor instability in prominences is to look at the formation of a bubble beneath a prominence model by flux emergence. From this the subsequent development of plumes as a result of the instability can be investigated in a selfconsistent fashion.

\subsubsection{How does the partially ionised plasma of prominences play a role in the development of this instability?}

The ionisation degree of prominences, which is the fraction of the prominence atoms that are ionised, is approximately 0.1 . This means that the majority of the fluid in a prominence is made up of neutral atoms, but one of the key forces in the support and dynamics of that fluid is the Lorentz force. Though the neutral particles do not directly feel the Lorentz force, those particles do collide with the ionised particles, which are feeling the Lorentz force. It is these collisions that allow the neutral component of the fluid to feel the magnetic forces.

There are a number of ways used to model the influence of partial ionisation on an MHD system, the most common being the single fluid approximation that uses a modified Ohm's law. The modification is that an extra diffusive term is added, known as ambipolar diffusion, to the induction equation. This term models the diffusion of the magnetic field through the predominantly neutral fluid. The inclusion of this term results in the induction equation becoming (e.g. Singh and Krishan 2010)

$$
\frac{\partial \mathbf{B}}{\partial t}=\nabla \times\left(\mathbf{u} \times \mathbf{B}-\eta \mathbf{J}+\frac{1}{c v_{\mathrm{IN}} \rho_{I}}(\mathbf{J} \times \mathbf{B}) \times \mathbf{B}\right) .
$$

The third term on the right-hand side of the equation is the ambipolar term, where $\rho_{I}$ is the ion density and $v_{\mathrm{IN}}$ is the ion-neutral collision frequency. There is some observational evidence that the drift between ions and neutral can be detected observationally in prominences (Khomenko et al. 2016) highlighting its potential dynamic importance.

Using this approximation, Khomenko et al. (2014) performed 2.5D simulations of a stratified cold layer supported above a hot layer with the magnetic field strength constant and set horizontally at an angle to the plane of the simulation. In the linear regime of the instability, the addition of ambipolar diffusion was found to result in the system always being unstable, whereas in an ideal MHD setting magnetic tension was found to suppress the instability on some scales. In the nonlinear regime of the instability, they found that the growth rate was increased by approximately $50 \%$ when ambipolar diffusion was included. The inclusion of ambipolar diffusion was also found to result in an increase of heating in the prominence material by a few tens of percent. Figure 12 shows the neutral and ion momentum, highlighting how different fluids are moving in different regions.

A more in-depth investigation into the influence on the linear instability by the presence of partially ionised plasma was performed by Díaz et al. (2012) and Díaz et al. (2014). Díaz et al. (2014) uses a single fluid model, though retaining more 

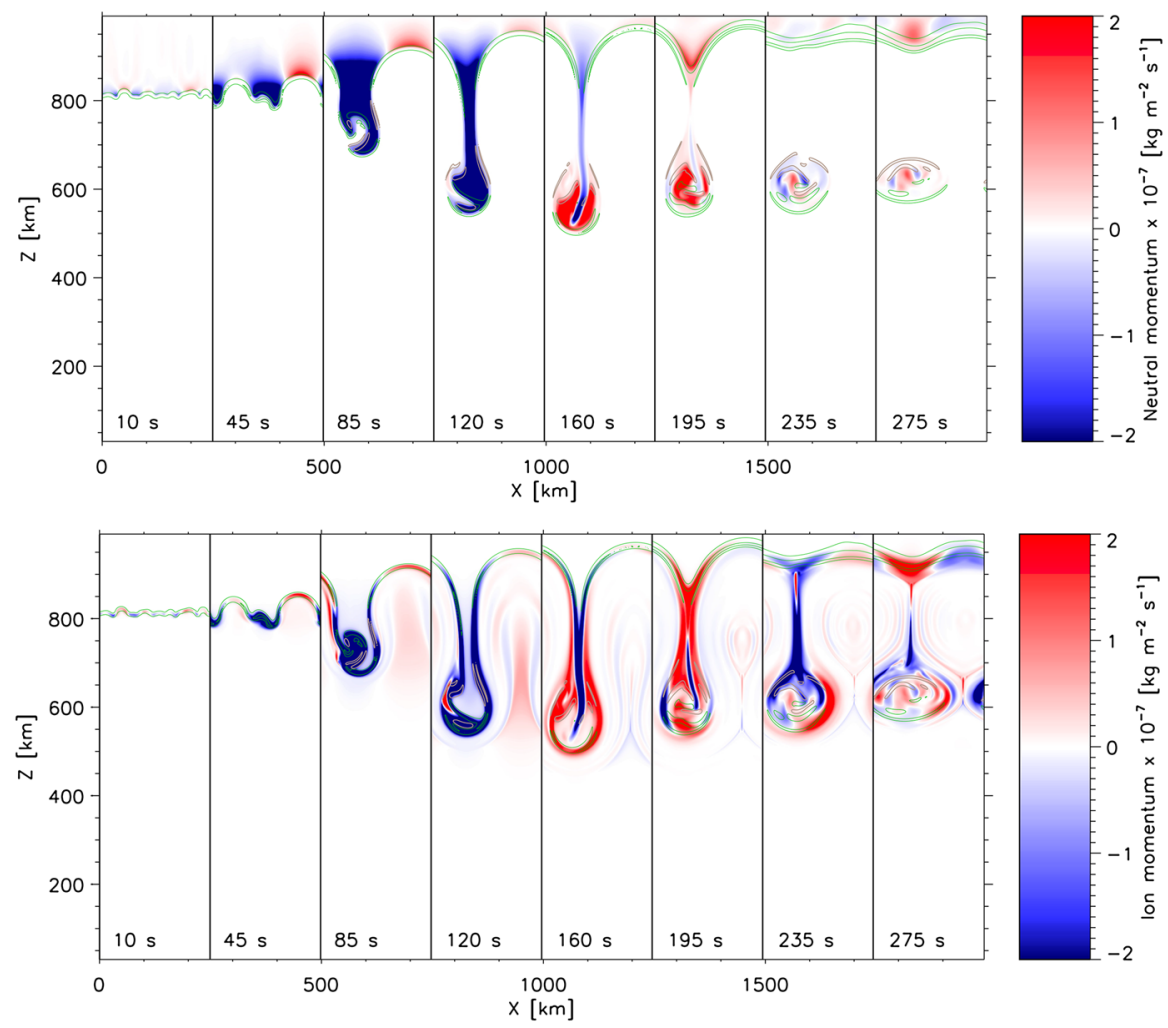

Fig. 12 Neutral and ion momentum as a result of plume evolution by the magnetic Rayleigh-Taylor instability. Credit: Khomenko et al. (2014), reproduced with permission (C) ESO

terms in the modified Ohm's law than in Eq. 19, to investigate the magnetic Rayleigh-Taylor instability finding that the magnetic field is not able to suppress the instability because the neutrals can slip across the magnetic field. Díaz et al. (2012) investigated the growth of the magnetic Rayleigh-Taylor instability in the classic uniform density layers with a compressible two-fluid model. The key result was that the inclusion of ion-neutral effects reduced the growth rate of the instability, making it more compatible with the observed lifetime of prominence threads.

\subsection{Other models}

There have been a number of other models proposed to explain the formation of prominence plumes. Here we look at these models and how they fit with the observed plumes. 


\subsubsection{Kelvin-Helmholtz, modes}

Ryutova et al. (2010) suggested that not all the plumes may be created by the magnetic Rayleigh-Taylor instability, but that the Kelvin-Helmholtz instability, in particular negative energy waves, could drive some of the plumes formed. This was motivated by the strong shear flows that were present at the bubble-prominence boundary in some cases. The dispersion relation for this case is presented in Eq. 16 of this paper, though the case used in Ryutova et al. (2010) did not include the shear of the magnetic field. It is the authors opinion that these two instabilities often work together, and so it is hard to truly separate them, but the existence of observational evidence for shear flows in a few cases means that this should not be ignored. The calculation of the perturbation energy in Ryutova et al. (2010) did not include the contribution from gravity, which as we have seen in Sect. 3 can change not only the instability threshold, but also the wave frequency. It would be very interesting to reperform their calculation including the effect of gravity in the estimate.

One question that deserves more investigation would be what is causing the shear flows. Based on the concept of emerging flux to form the bubbles, and that this flux may be twisted, then we can put forward one hypothesis as to why bubble-driven shear flows may exist. Observationally it has been shown that sunspots rotate (e.g. Brown et al. 2003). In numerical experiments, where the scale of the emerging flux region is by necessity smaller than a full active region, the rotation rate is normally larger (e.g. Sturrock et al. 2015). This rotation of the region, treating the bubble as a small region of twisted emerging flux, as it emerges would result in flows in the bubble but there would be no flows in the prominence, resulting in shear flows.

\subsubsection{Other buoyancy modes}

There are other buoyancy instabilities than the magnetic Rayleigh-Taylor instability, and Hurlburt and Berger (2012) suggested that double-diffusive convection, where the two diffusions are magnetic and thermal, may be their driver. The condition for this instability is

$$
\frac{\partial V_{\mathrm{A}}^{2}}{\partial z}<0,
$$

that is the Alfvén speed decreases with height. As the plumes form from something approaching a contact discontinuity suggests a surface mode, which can grow very quickly due to the large density difference giving a large buoyancy force, and not an internal mode. However, as with other magneto-buoyancy instabilities as discussed in Sect. 2.1, the possibility that the conditions for these instabilities are met in prominences would explain a lot of the motions that are observed in prominences.

\subsubsection{Magnetic reconnection}

Quite different to these models, Dudík et al. (2012) proposed a model where current sheets form at the boundary between the bubble and prominence magnetic field. 
They suggested that reconnection in these current sheets results in the plume formation, where the plumes are formed of packets of reconnected flux. The idea of reconnection driving plume formation is also put forward in Gunár et al. (2014). The presence of the separatrix between the bubble and the prominence in the model of Dudík et al. (2012) led the authors to hypothesis that only reconnection, and not the Rayleigh-Taylor instability, could be used to explain plumes because without reconnection magnetic flux cannot cross a separatrix. However, the RayleighTaylor instability is able to form plumes by deforming the separatrix as can be understood from the linear analysis of the magnetic Rayleigh-Taylor instability in a sheared magnetic field (see Eq. 14 or Ruderman et al. 2014; Hillier 2016) circumnavigating this issue.

Shen et al. (2015) observed a bubble using the New Vacuum Solar Telescope (NSVT) and AIA on the 24 May 2014 (the inference of the magnetic field for this prominence was presented in Levens et al. 2016 where around the rim of the bubble was one of a number of places that displayed $60 \mathrm{G}$ patches of magnetic field), finding repeated flaring activity at the base of a bubble and well as oscillations of the bubble boundary at periods of 3 and $5 \mathrm{~min}$. They suggest that this flaring activity results in the collapse of a bubble. The hypothesis they put forward is that reconnection between the prominence magnetic field and that of the bubble result in the collapse and this reconnection heats the bubble. However, the authors were not able to rule out the Rayleigh-Taylor instability because it would result in the observed dynamics at the bubble boundary.

As a theory, unlike the magnetic Rayleigh-Taylor instability, there has not been an explanation as to how the reconnection model can explain most of the observed features. If we think of the observations of the plumes we need a theory to explain: the fact the motions are dominated by vertical flows, that the plumes rising upward are much larger than the small spikes of material that fall down (e.g. see panel B of Fig. 5), or in fact why the difference in plume size forms at all. At present, no model presented provides a reason as to why the plume dynamics are only observed in quiescent prominences, as it could be expected that the reconnection hypothesis should work equally for all magnetic field strengths. For this model to be used to understand plume formation, it is necessary for it to be developed to explain the observed plume features.

There is one clear and understandable prediction from any model of reconnection between emerging magnetic flux and an ambient magnetic field, which can be made based on the long history of numerical studies of this topic. This is the formation of jets ejected parallel to the component of the magnetic field that reconnects (Shibata et al. 1992). In the case of a prominence and bubble interaction this would be roughly parallel to the interface between the bubble and the prominence and would be observed as a spray of the cool prominence material. It can be hypothesized that these flows would be observable in the cool lines used to observe the prominence as a jet of cool material ejected from the boundary (and not just flows of material along the boundary).

To summarise my opinion, it is likely to be possible that magnetic reconnection can occur in this setting, and if reconnection occurs it may alter the stability criteria at the bubble-prominence boundary because of changes in the magnetic topology or 
by providing a velocity perturbation to the system to excite an instability. However, there is currently no theoretical work that suggests that reconnection could drive anything that looks like a plume.

\subsubsection{Comparison between models}

Of all the theories proposed to explain the formation of the plumes, the RayleighTaylor instability is the one that has been most tested. Not only do the linear and nonlinear theories agree with the observations, the nonlinear numerical simulations have been highly successful at reproducing the observed dynamics. The connection with Kelvin-Helmholtz modes, and other buoyancy modes, is clearly necessary at times, and this could provide an interesting place for developing further understanding. Magnetic reconnection, however, does not appear to be able to explain the plumes as they are observed. This does not mean that magnetic reconnection cannot happen at the bubble-prominence boundary, it just is in the opinion of the author more likely to create sprays of cool prominence material from this region. It would be interesting to re-analyse the current observations to see if this happens and how regularly it occurs.

\subsection{Fragmenting eruption and the magnetic Rayleigh-Taylor}

The processes that fragmented 7 June 2011 eruption have also been attributed to the magnetic Rayleigh-Taylor instability. Innes et al. (2012) pointed out the morphological similarity between the fragmentation of a bubble and that of a splash of liquid with non-negligible surface tension, the dynamics of which are known to be driven by the Rayleigh-Taylor instability (Allen 1975). In the case of the eruption, the role of surface tension is replaced by magnetic tension.

Innes et al. (2012) also calculated the acceleration of the bubbles during the fragmentation. Fitting a $t^{2}$ curve to the motion, they found an acceleration of $\sim 140 \mathrm{~m} \mathrm{~s}^{-2}$. This is less than the $274 \mathrm{~m} \mathrm{~s}^{-2}$ of the surface gravity so is significantly less than free fall. This would be consistent with a most unstable undular mode of the system where the growth rate becomes (Hillier 2016)

$$
\sigma^{2}=\frac{1}{2} A g k_{\text {unstable }}
$$

i.e. gravity is effectively reduced by a factor of $1 / 2$ by magnetic tension. However, in this late stage of the evolution of the instability it is unlikely that such linear relations can still be accurately applied and the height above the solar surface is likely to have significantly reduced gravitational acceleration from its surface value (Carlyle, private communication).

One interesting comparison that can be made here is to the nonlinear evolution of the Parker instability in a stratified atmosphere. As discussed in Chapter 3, Section 2.2 of Tajima and Shibata (2002) and Shibata et al. (1990), the emergence of magnetic field into the solar atmosphere undergoes four different stages. First there is the linear instability, which grows exponentially. Then comes a self-similar 
exponential expansion phase from the nonlinear instability. After that comes another self-similar phase of either a power law expansion with the rise velocity $\left(V_{z}\right)$ given as $V_{z} \approx \alpha z / t$ or a force-free expansion, which follows the same trend. Then if there is a hot corona or an overlying magnetic field then the expansion would stop. The second of the self-similar phases, where there is a power-law expansion, would produce the $t^{2}$ behaviour observed. Note that this behaviour is similar to that of the thickening of the mixing layer by the incompressible Rayleigh-Taylor instability as given in Equation 17.

\section{Using the magnetic RT to determine the magnetic field strength of a prominence}

With strong evidence supporting the Rayleigh-Taylor instability as the explanation for the formation of the plumes, it has been possible to use what we understand about the instability to infer the physical conditions of the observed prominences. In this section I will review the various attempts that have been made to do this.

\subsection{Linear growth rate of the $R T$ instability}

The use of a linear dispersion relation to estimate the strength of the magnetic field in the solar atmosphere is one that is often applied in solar physics. The key difference here is that instead of use a wave for the inversion (c.f. Section 3.3 of Nakariakov and Verwichte 2005) an instability is used.

The key observables that can be applied to determine the magnetic field strength from Eq. 12 are the wavelength observed and the growth rate of the instability. It is exactly these two that Ryutova et al. (2010) observed in 30 November 2006 prominence, finding a wavelength of $1.2 \times 10^{8} \mathrm{~cm}$ and a growth rate of $\sim 8 \times 10^{-3} \mathrm{~s}^{-1}$. Taking an angle between the magnetic field and the wave vector of $\theta=85^{\circ}$ and a field strength of $6 \mathrm{G}$ the observed wavelength would give the observed growth rate.

This approach is not without its problems. First, the growth rate is very difficult to determine observationally as the linear stage of the instability ends when the plume is about as tall as it is wide (e.g. Hillier 2016). In the paper, the plume height is measured at only two times to determine the growth rate, so not only would this have some intrinsic error, a large range of curves that are not exponential curves could also be fitted to these points. That is to say, this may not be the growth rate of the linear instability. The wavelength also can be problematic because of projection effects, that is to say that the observed wavelength is the lower limit for the actual wavelength (see Sect. 5.2 for a possible way to determine the projection effects in the system). Finally, even if both of these have been determined to a high level of accuracy, the dispersion relation only allows you to determine $B \cos \theta$, where $\cos \theta$ is the angle between the wave vector and $\mathbf{B}$, and not the value of $B$ itself.

To reduce the difficulties set out above, to estimate the magnetic field strength associated with the fragmenting eruption discussed in Sect. 2.5, Innes et al. (2012) used the critical wavelength to estimate the Alfvén speed in the dense material. 
They found speeds of $47 \mathrm{~km} \mathrm{~s}^{-1}$. On the same set of observations, Carlyle et al. (2014) employed a most unstable mode analysis of the magnetic Rayleigh-Taylor instability to determine the field strength. This is similar to the method used by Hester et al. (1996) to investigate the magnetic field of the filamentary structure observed in the Crab Nebula. To do this, first they took the derivative of Eq. 12 with respect to $k$, and then assuming that a pure undular mode is being observed they showed that the strength of the magnetic field $(B)$ can be estimated by

$$
B=\sqrt{\frac{g \lambda_{u}\left(\rho_{+}-\rho_{-}\right)}{2}},
$$

where $g$ is the constant gravity, $\lambda_{u}$ is the wavelength of the undular mode (in this case the observed wavelength) and $\rho_{+}$and $\rho_{-}$are the densities of the high and low density regions. Using the observed density and wavelengths they obtained from their analysis, they estimated a field strength of $\sim 1 \mathrm{G}$.

There are still issues associated with this method. One being that Eq. 12 for the growth rate is unbounded as a result of the problem being ill-posed. Carlyle et al. (2014) noted this problem and identified that if the most unstable undular mode is taken, as was done in the paper, this would give an estimate of the weakest component of a sheared magnetic field. Therefore, though this method would not be able to determine the exact field strength, it would provide a lower estimate for the magnetic field strength.

The growth rate for the sheared field case was determined by Ruderman et al. (2014), though more specific cases had been investigated (e.g. Goedbloed and Poedts 2004, Equation 6.612). Hillier (2016) suggested the use of the dispersion relation given in Eq. 14 for application to observations due to its applicability to situations where the plasma $\beta$ is low. When reapplying this equation using the method of Carlyle et al. (2014) to their results this implies that, as they originally suggested, the lower limit of the magnetic field strength is $1 \mathrm{G}$.

Ruderman (2017) applied the compressible magnetic Rayleigh-Taylor growth rate in the case of a sheared magnetic field to the observed plume widths and growth rates of Ryutova et al. (2010). Taking the same strength of magnetic field of $6 \mathrm{G}$, they were able to show that the observations are consistent with a small shear between the prominence and bubble magnetic field of $<15^{\circ}$ (with a $180^{\circ}$ ambiguity).

Berger et al. (2017) used measured plume characteristics and inference of the shear flows at the prominence-bubble boundary to evaluate the magnetic field associated with observed plume formation. Using the equation for the growth of the magnetic Rayleigh-Taylor-Kelvin-Helmholtz instability and constraining the solution by the observed growth rate, the wavelength and the bubble size (the latter being used to approximate the effects of line-tying of the magnetic field in the model). The resulting solution found for prominence densities gave a magnetic field strength of approximately $10 \mathrm{G}$. As the observation was of a polar crown prominence, the small angle found between the magnetic field and the line-of-sight is consistent with the prominence magnetic field being highly sheared with respect to a reference potential field calculated from the photospheric field associated with the prominence. 


\subsection{Nonlinear plume flows}

Another method to use the presence of these plumes to determine the strength of the magnetic field was presented by Hillier et al. (2012c). This method is based on the flows that should be set up around the plume head as it moves through the prominence (i.e. those observed by Berger et al. 2011; Orozco Suárez et al. 2014), and the subsequent compression of the prominence material by these flows. Using these flows, Hillier et al. (2012c) was able to create a method to estimate the compression from observations and then use this to estimate the plasma beta of the prominence medium.

To develop the model, a set of key observational characteristics of the plumes in their nonlinear regime were provided.

1. The plumes rise at an approximately constant velocity (Berger et al. 2010).

2. The plumes have an elliptical head (Berger et al. 2010, and also see Fig. 4).

3. The intensity of the prominence material at the top of the plume is greater than the average prominence intensity (Hillier et al. 2012c, and also see Fig. 4).

4. Flows of material can be observed along the head of the plume (Berger et al. 2011; Orozco Suárez et al. 2014).

5. The prominence magnetic field is mainly horizontal (e.g. Schmieder et al. 2013).

To these, one characteristic of the magnetic Rayleigh-Taylor instability is added, that is the fact that the magnetic Rayleigh-Taylor instability creates structures aligned with the magnetic field (Hillier et al. 2012a).

The authors applied the following method to reduce the flow rise to a simple model. They first removed the plume stem, treating it as a rising ellipse in the prominence material. As this ellipse is moving at constant velocity through (what can be roughly assumed to be) a constant medium, a shift in reference frame gave a stationary ellipse in a constant flow. Then using the field-aligned structure expected for the plume they deduce that the problem can be viewed as being similar to an elliptical cylinder in a constant flow. Finally they performed a conformal transform of the coordinate system to change to a circular cylinder creates a situation analogous to that of a constant flow around a circular cylinder.

For simplicity, they made the assumption that the plasma $\beta$ is approximately uniform. Based on this assumption, and modifying the derivation for a compressible flow around a circular cylinder (van Dyke 1975) where the lengthscale has been normalised by the cylinder radius, the density and velocity are normalised by their upstream values, and cylindrical polar coordinates with the origin at the centre of the cylinder are used, they derived the following equations:

$$
\begin{gathered}
\rho=\left(1-\frac{\gamma-1}{2} M_{*}^{2}\left(v_{r}^{2}+v_{\theta}^{2}-1\right)\right)^{1 /(\gamma-1)} \\
v_{r}(r, \theta)=\left(1-\frac{1}{r^{2}}\right) \cos \theta-M_{*}^{2}\left[\left(-\frac{13}{12} \frac{1}{r^{2}}+\frac{3}{2} \frac{1}{r^{4}}-\frac{5}{12} \frac{1}{r^{6}}\right) \cos \theta\right. \\
\left.+\left(\frac{1}{4} \frac{1}{r^{4}}-\frac{1}{4} \frac{1}{r^{2}}\right) \cos ^{3} \theta\right]+O\left(M_{*}^{4}\right)
\end{gathered}
$$




$$
\begin{gathered}
v_{\theta}(r, \theta)=-\left(1+\frac{1}{r^{2}}\right) \sin \theta+M_{*}^{2}\left[\left(\frac{13}{12} \frac{1}{r^{2}}-\frac{1}{2} \frac{1}{r^{4}}+\frac{1}{12} \frac{1}{r^{6}}\right) \sin \theta\right. \\
\left.-\left(\frac{1}{12} \frac{1}{r^{4}}-\frac{1}{4} \frac{1}{r^{2}}\right) 3 \cos ^{2} \theta \sin \theta\right]+O\left(M_{*}^{4}\right) \\
M_{*}=\sqrt{\frac{\gamma \beta_{\infty}}{\gamma \beta_{\infty}+2(\gamma-1)}} M_{\infty},
\end{gathered}
$$

where $\gamma$ is the ratio of specific heats, $M_{\infty}$ is the upstream hydrodynamic Mach number of the flow and $\beta_{\infty}$ is the upstream plasma $\beta$. Using Eq. 23 they investigated the compression around a plume head. The density distribution as produced by Eq. 23 is shown in Fig. 13.

This model provides a density distribution, and to compare this to the observations it must be related to the prominence intensity. The prominence intensity is dominated by scattering, giving the intensity as a function of the column density. For simplicity in Hillier et al. (2012c) they take the intensity as linearly proportional to the density, though taking it as proportional to the square of the density is likely to be more appropriate. Hence, the bright top of the plume would imply that there has been an increase in the density through compression of the prominence. Note that as the flow distributions are also given, it is possible to calculate the angle of the tube to the line-of-sight using Doppler velocity measurements.

Using the plume observations shown in Fig. 4, they determined the Mach number of the rising plume and the plume dimensions. This prominence was observed on the northwest solar limb on 3 October 2007 observed by the SOT with the Ca II H filter at a cadence of $30 \mathrm{~s}$. The velocity of the plume was found to be approximately constant, with velocity and the velocity error calculated to be $v_{\text {plume }}=12.3 \pm 0.6 \mathrm{~km} \mathrm{~s}^{-1}$ giving a Mach number of the flow of

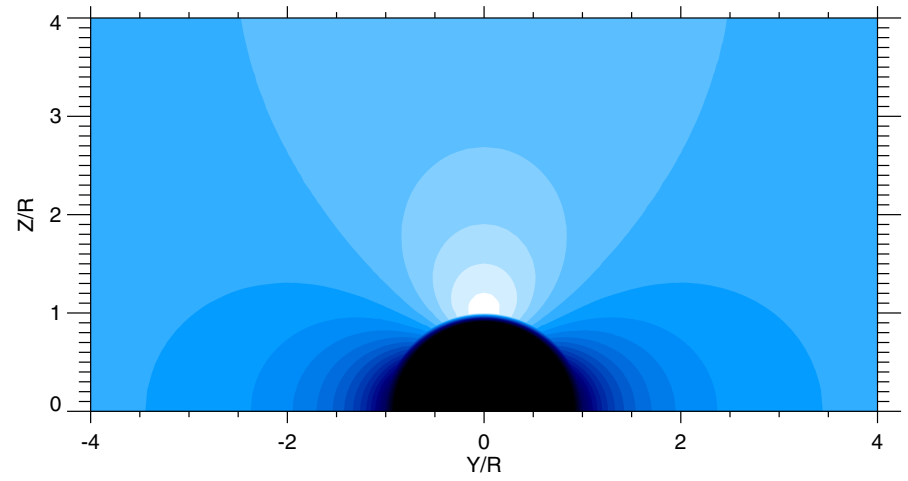

Fig. 13 Contour plot of the density distribution used to model the plume head. Reproduced with permission from Hillier et al. (2012c) 
$M_{\infty}=1.12 \pm 0.05$. From fitting the intensity at the plume head, the value of $M_{*}$ was estimated and this was used to solve for the plasma beta of the prominence, where rearranging Eq. 26 to find $\beta_{\infty}$ is given by

$$
\beta_{\infty}=\frac{2(\gamma-1)}{\gamma} \frac{M_{*}^{2}}{M_{\infty}^{2}-M_{*}^{2}} .
$$

Assuming the range $\gamma=1.4-1.7$, they found a plasma $\beta$ in the prominence of $\beta_{\infty}=0.47 \pm 0.079$ to $1.13 \pm 0.080$. Therefore, though the field strength is not possible to be determined, the plasma beta can be estimated from the plume dynamics.

One limitation of this model, and with it one area the theory could be improved, is the connection between the density distribution and the intensity. Hillier et al. (2012c) simply assume that the intensity is directly proportional to the density. It would be very interesting to combine the model of Hillier et al. (2012c) with the $\mathrm{H} \alpha$ intensity model of Heinzel et al. (2015) to provide more accurate estimates of the plasma $\beta$ of a prominence though significantly more realistic intensity modelling.

\section{Connections with other astro-, space and plasma physical systems}

Solar prominences are not the only places where the magnetic Rayleigh-Taylor instability can occur. In the following section, I will review some of these instances and look at the connections they hold to the magnetic Rayleigh-Taylor instability in prominences.

\subsection{The solar atmosphere and the heliosphere}

The emergence of magnetic flux into the solar atmosphere, itself associated with a magnetic Rayleigh-Taylor like mode in the Parker instability (e.g. Shibata et al. 1989), has been hypothesized to become Rayleigh-Taylor unstable. Isobe et al. $(2005,2006)$ studied the 3D emergence of a magnetic flux sheet. They found that as the magnetic flux emerged, dense material would collect at the apex of the emerging flux, creating a Rayleigh-Taylor unstable situation where dense material is supported above light material. The instability developed, dominated by interchange modes, creating field-aligned filamentary structure. The creation of filamentary structure resulted in the formation of current sheets which led to magnetic reconnection, this coincided with an inverse cascade in scales perpendicular to the magnetic field. The Rayleigh-Taylor instability was also cited as being an important part of the two-step emergence process for magnetic flux (Toriumi and Yokoyama 2012). However, Arber et al. (2007) found that the inclusion of ion-neutral interaction through a modified Ohm's law, see Eq. 19, in a 3D flux emergence simulation suppressed the development of the instability by allowing the neutral material to slip across the magnetic field. Therefore, more work is required to 
understand if this instability can really occurring as a result of emerging magnetic flux.

Supra-arcade downflows (SADs; e.g. Savage et al. 2012) are turbulent downflows that develop above flare arcades (McKenzie 2013). One interpretation given to SADs is that they are created by the Rayleigh-Taylor instability at the head of the reconnection jet (Innes et al. 2014). Simulations by Guo et al. (2014) confirm that this mechanism can produce dynamics similar to the observed downflows.

The rise of regions of depleted plasma against the gravitational field during the equatorial spread F phenomenon in the Earth's ionosphere is an important process that can influence radio signal propagation. This has been interpreted as the occurrence of the magnetic Rayleigh-Taylor instability in a low-beta magnetic plasma environment (Kelley et al. 1976; Takahashi et al. 2009). The dynamics of the rising equatorial plasma bubbles simulated by Yokoyama et al. (2014) are remarkably similar to those of the prominence plumes.

Changes in the heliosphere can also drive the instability at both the magnetopause and the heliopause. Gratton et al. (1996) described how variations in the solar wind speed can lead to expansion and contraction of the magnetopause, resulting from changes in dynamic pressure, could lead to the development of the Rayleigh-Taylor instability. In the expansion phase of the magnetopause, there is an effective gravity that could potentially drive this instability. A similar physical process can also happen at the heliopause as a result of changes due to the solar cycle (Borovikov and Pogorelov 2014).

The Rayleigh-Taylor instability in magnetic flux emergence, the spread-f phenomenon, and the magnetopause all share a common magnetic field geometry, i.e. the arched magnetic field. I have already presented the potential connections between flux emergence by the Parker instability and the nonlinear state of the fragmenting eruption in Sect. 4.3. What is most striking is the incredibly similar morphology that can be found across these phenomena. Figure 14 gives a comparison between observations of supra-arcade downflows, a simulation of equatorial plasma bubbles and observations of prominence plumes, where a remarkable similarity exists.

The work of Isobe et al. $(2005,2006)$ presents a situation that can be imagined to be very similar to the current hypothesis for magnetic flux emerging beneath a prominence and as such the prominence material collecting above it and the system ultimately becoming Rayleigh-Taylor unstable. It shows us that as with the equatorial spread $\mathrm{F}$ phenomenon, for example, when the magnetic Rayleigh-Taylor instability occurs at the top of a magnetic bubble, the rising plumes it produces will relate to the loop-like expansion of the magnetic field of that bubble. This would likely imply that on the large scale, the plumes are made up of field lines with downward curvature as with the spread-f plumes (see Fig. 4 of Yokoyama et al. 2014), but locally in the plume the field may be flattened, or become locally upwardly curved, by the interaction with the prominence (e.g. note the flattening of the field lines in Isobe et al. 2006). 

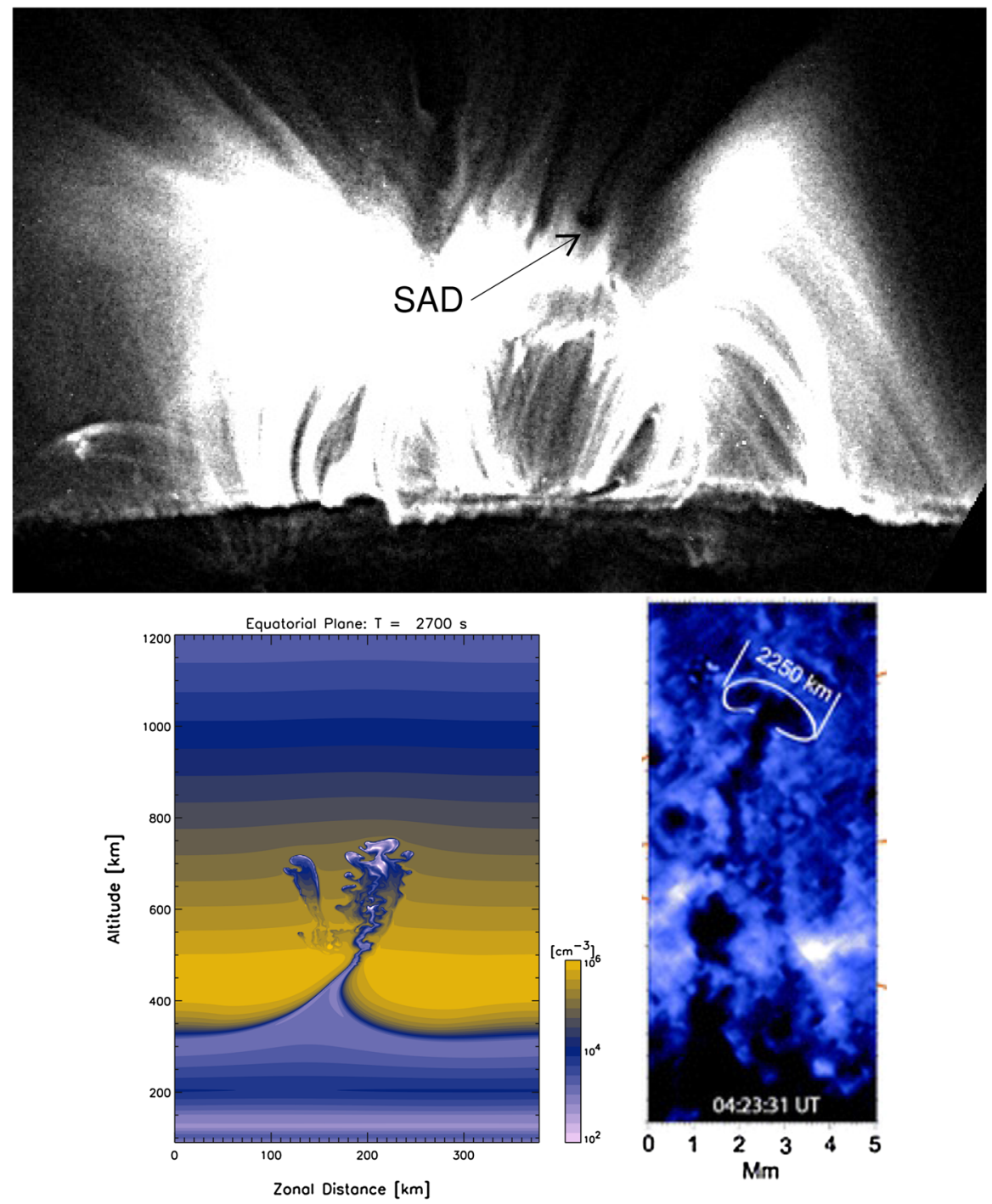

Fig. 14 (Top) SADs observed by AIA in the $131 \AA$ bandpass on 27 January 2012, (Left) Equatorial plasma bubbles (Courtesy of Dr. T. Yokoyama (NICT) from Yokoyama et al. 2014 (C) American Geophysical Union) and (right) a quiescent prominence plume (from Figure 2 of Berger et al. 2008) (C)AAS. Reproduced with permission

\subsection{Plasma and fluid experiments}

Moser and Bellan (2012) presented experimental results of the development of the kink instability in a plasma jet. They found that for jets filled with material of sufficient mass, the lateral acceleration of the jet material by the kink instability provided an effective gravity and this allowed the magnetic Rayleigh-Taylor instability to grow. In a similar process to that seen in Isobe et al. (2006), the 
nonlinear development led to magnetic reconnection. Zhai and Bellan (2016) developed linear theory to describe the growth of the hybrid of the current driven kink instability and the gravitationally driven magnetic Rayleigh-Taylor instability. They found that the combined instability grew significantly faster than either of the two individual instabilities by themselves. Though it can be argued that in the jets the Rayleigh-Taylor instability is purely a parasitic instability, as the effective gravity is created by the growth of the kink instability, this work does have particular relevance for the understanding of how the magnetic Rayleigh-Taylor instability can develop in the flux-tube geometry expected to be associated with prominences and how it may connect with the global stability of prominences. One area this study is of importance is in the understanding of how curvature of the magnetic field can change the growth rate of the system. Because they found that the combined instability increased the growth rate, this can at least provide some evidence that the curved magnetic field of a prominence may help the development of the magnetic Rayleigh-Taylor instability in that setting.

Magnetic fields have also been used in experiments of the Rayleigh-Taylor instability by Scase et al. (2016), in this case using the magnetic field to create the density inversion through diamagnetic and paramagnetic effects, to investigate the Rayleigh-Taylor instability in a rotating system. They found that the growth rate could be reduced, and in some cases stabilised, by rotation. This brings to mind the recent discovery of tornado prominences (e.g. Orozco Suárez et al. 2012; Su et al. 2014), hypothesized to be rotating columns of plasma and magnetic field (Luna et al. 2015), and could have important consequences for understanding their stability.

\subsection{Astrophysical systems}

Supernovae are one well-known example of where the Rayleigh-Taylor instability can develop. Chevalier et al. (1992) performed a linear perturbation analysis on the self-similar solution of Chevalier (1982) for the interaction of stellar ejecta interacting with an external medium finding that it is unstable to the hydrodynamic Rayleigh-Taylor instability in supernovae like cases. In this case, the instability was driven by an effective gravity produced by the interaction of the two media. Hachisu et al. (1992) discussed how the development of the Rayleigh-Taylor instability would result in element mixing in supernova explosions.

Hester et al. (1996) compared the observational characteristics of the Crab Nebula, see Fig. 15, with simulations of the magnetic Rayleigh-Taylor instability performed by Jun et al. (1995), showing that the magnetic Rayleigh-Taylor instability could well describe the observed filamentary structure. Jun and Norman (1996) found that the evolution of the Rayleigh-Taylor instability, and the KelvinHelmholtz instability it excited, could locally increase the strength of the magnetic field by a factor of $\sim 60$. Jun et al. (1996) suggested that the Rayleigh-Taylor plumes could protrude through the forward shock, suggesting that these could be the sites of strong radio and X-ray emission. Recent axisymmetric simulations of the Crab Nebula by Porth et al. (2014), using adaptive mesh refinement to provide high 


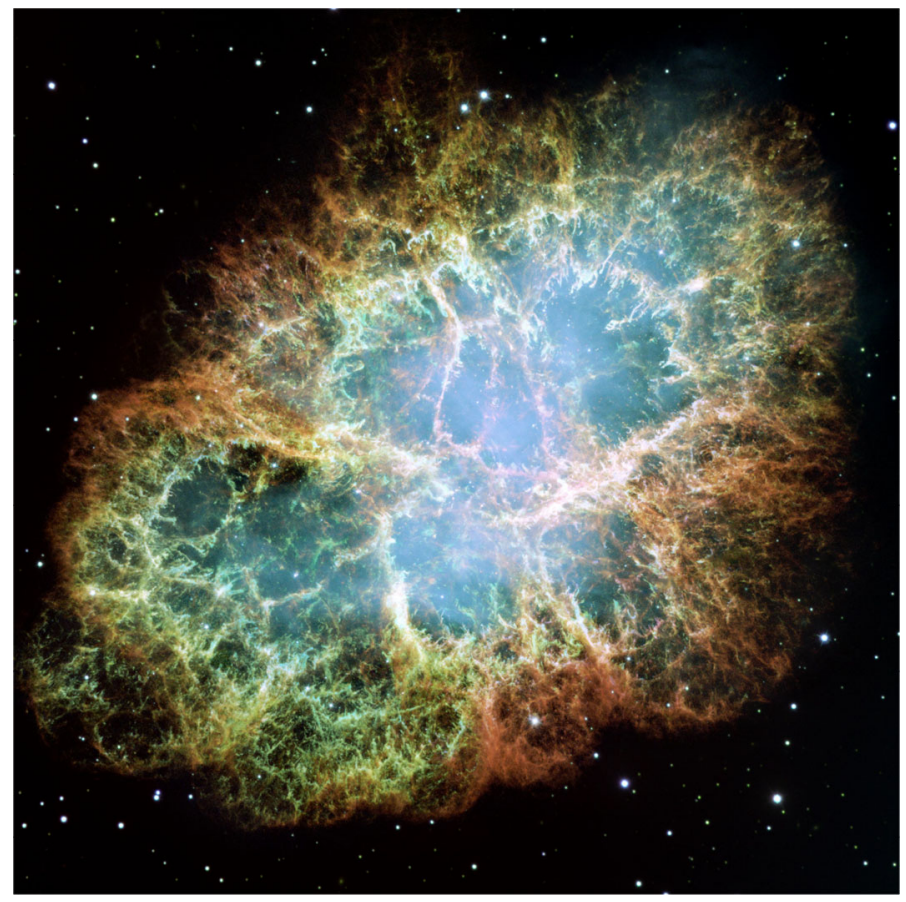

Fig. 15 Filamentary structure of the Crab Nebula. Image credit: NASA, ESA, J. Hester, A. Loll (ASU)

resolution, found that the magnetic field is insufficient to suppress the growth of the instability.

An effective gravity can come in many forms. Matsumoto and Masada (2013) performed special relativistic hydrodynamic simulations of a relativistically hot, relativistically fast astrophysical jet finding that the inertia force (that acts to halt the lateral expansion of the jet) provides an acceleration that can drive the RayleighTaylor instability on the condition that the effective inertia ratio of the jet to the external medium is less than one. They suggested that this instability, combined with the Richtmyer-Meshkov instability that feeds off the Rayleigh-Taylor fingers, could be a mechanism to destroy the structure of relativistic jets.

The interchange instability has been proposed as a method to aid accretion onto magnetised stars (e.g. Spruit et al. 1995). In 3D MHD simulations by Kulkarni and Romanova (2008) investigated the development of the magnetic Rayleigh-Taylor instability at the accretion disk magnetosphere boundary. They found that the instability occurred when there was a misalignment of less than $30^{\circ}$ between the magnetic and rotation axis. Spruit and Taam (1993) proposed that for strong stellar magnetic fields, the system would become unstable as mass piled up on the boundary resulting in accretion occurring in a cyclic fashion. This bares some interesting analogies to the formation of prominence plumes, but instead of having mass build up until instability, it could be seen as the change from the stronger fields 
of active region prominences to those of the weaker field strength of quiescent prominences that allows instability.

Cattaneo and Hughes (1988) investigated the breakup of a magnetic layer in the solar interior by the interchange instability. Due to the top heavy nature of isothermal layer of magnetic flux, it becomes unstable to the Rayleigh-Taylor instability. This result was found for both static and uniformly rotating setups. In the nonlinear evolution of the instability, the shear flows created by the instability gave rise to the Kelvin-Helmholtz instability which created localised vortices. If vortices from the same plume interacted they worked to drive the plume further upward, but when vortices from different plumes interacted they would work to drive the two plumes downward.

Though the parameters of solar prominences are rather different from those of the phenomena discussed here, there are still strong comparisons. The clear comparison to prominences comes from the fragmenting eruption which, as pointed out by Carlyle et al. (2014), is remarkably morphologically similar. This has been used to develop further the application of instabilities to determine field strengths, and understand the inherent problems this method possesses.

\section{Summary}

In this paper, we have looked at the physics behind the formation of plumes in prominences and the fragmentation of a prominence eruption. The physical process that appears to be behind these observations is the magnetic Rayleigh-Taylor instability.

The formation of the plumes in quiescent prominences relates to the formation of a low-density bubble beneath the prominence. This then results in the dense prominence material sitting above that of the low-density bubble. The boundary between these two then goes unstable to the Rayleigh-Taylor instability, which creates plumes that rise at a constant velocity. These plums often breakup, turbulently mixing into the prominence material. The observations of quiescent prominences show that these plumes are a very common feature. Numerical simulations, in both local and global prominence models, have been very successful in reproducing the characteristics of the observed plumes through the nonlinear stages of the magnetic Rayleigh-Taylor instability. For models of low-lying prominences, which are more like the observed height of intermediate and active region prominences, increasing the shear of the prominence magnetic field with reference to a potential field solution (which is akin to increasing the field line strength to length ratio) resulted in reduction in the growth of the instability. The possible changes to the model to make them more unstable to the Rayleigh-Taylor instability (including making the prominence taller and making sure a sharp density transition exists) are predictable from theory and would result in the prominence model becoming closer to the conditions for observed plume formation. However, there is still improvement that needs to be made in the models by making them more like the observed prominences and including their interaction with bubbles to fully understand the nature of plume formation. 
The fragmenting eruption happens through a very similar physical process. The dense prominence material is falling through the low density corona, creating the required density inversion for a system to become Rayleigh-Taylor unstable. In this fragmentation, the separation of the plumes consistently developed at $10^{9} \mathrm{~cm}$ with smaller perturbations damping away (Innes et al. 2012). The nonlinear regime of the instability displays the self-similar evolution growing as $t^{2}$ as theoretically predicted. Not only does this connect with the thickening of the mixing layer due to the Rayleigh-Taylor instability, it also connects to the nonlinear regimes of the Parker instability.

Using the linear and nonlinear conditions, it has been possible to determine information about the magnetic field strength of the prominences. Ryutova et al. (2010) found field strengths of $6 \mathrm{G}$ in a prominence by applying the linear instability relation to prominence plumes, and Hillier et al. (2012c) used the nonlinear plume flows in a separate prominence to estimate a plasma $\beta$ of $\beta \sim 0.5$. For the fragmenting eruption, the linear instability suggested field strengths in the upper corona of $1 \mathrm{G}$ (Carlyle et al. 2014). Due to the inherent difficultly in measuring prominence field strengths, the ability to use the manifestations of this instability to estimate field strengths and directions is of great importance for developing our understanding of prominences.

There are many other examples of the magnetic Rayleigh-Taylor instability in astrophysics, space physics and plasma physics. However, due to the high quality of the prominence observations and the clear connections that can be drawn between the prominence examples and the other examples, it makes them especially important in developing our theoretically understanding of this instability by placing clear observational constraints on any theory. These other examples also feedback into our understanding of the prominence system, especially as they allow us to gain some understanding about how the magnetic Rayleigh-Taylor instability develops when magnetic fields are curved. This makes it an especially exciting time to be working on prominence physics.

Acknowledgements The author would like to thank the following people for their help and support. Prof. K. Shibata for first introducing me to this topic, Dr. T. Berger for the numerous discussions on this topic that have been key to developing my opinions into solid theory, Dr. J. Carlyle for helping me to understand the connection between the theory and the observations, and Dr. H. Isobe for many an insight into the physics of the Rayleigh-Taylor instability. The author would also like to thank the three anonymous referees for their insightful comments that greatly improved the content of this manuscript.

\section{Compliance with ethical standards}

Conflict of interest On behalf of all authors, the corresponding author states that there is no conflict of interest.

Open Access This article is distributed under the terms of the Creative Commons Attribution 4.0 International License (http://creativecommons.org/licenses/by/4.0/), which permits unrestricted use, distribution, and reproduction in any medium, provided you give appropriate credit to the original author(s) and the source, provide a link to the Creative Commons license, and indicate if changes were made. 


\section{References}

K. Ahn, J. Chae, W. Cao, P.R. Goode, ApJ 721, 74 (2010)

R.F. Allen, J. Colloid Surf. Sci. 51, 350 (1975)

J.-J. Aly, ApJ 746, 52 (2012)

P. Antolin, T.J. Okamoto, B. De Pontieu et al., ApJ 809, 72 (2015)

U. Anzer, Sol. Phys. 8, 37 (1969)

T.D. Arber, M. Haynes, J.E. Leake, ApJ 666, 541 (2007)

I. Arregui, R. Oliver, J.L. Ballester, Living Rev. Sol. Phys. 9 (2012)

A. Asai, T.T. Ishii, H. Isobe et al., ApJ 745, L18 (2012)

G. Aulanier, P. Demoulin, A\&A 329, 1125 (1998)

G. Aulanier, P. Démoulin, A\&A 402, 769 (2003)

T.E. Berger, R.A. Shine, G.L. Slater et al., ApJ 676, L89 (2008)

T.E. Berger, G. Slater, N. Hurlburt et al., ApJ 716, 1288 (2010)

T. Berger, P. Testa, A. Hillier et al., Nature 472, 197 (2011)

T.E. Berger, W. Liu, B.C. Low, ApJ 758, L37 (2012)

T. Berger, Nature of prominences and their role in space. Weather 300, 15 (2014)

T.E. Berger, A. Hillier, W. Liu, ApJ 850, 60 (2017)

A. Berlicki, S. Gunar, P. Heinzel, B. Schmieder, P. Schwartz, A\&A 530, A143 (2011)

J.W.S. Blokland, R. Keppens, A\&A 532, A93 (2011)

J.W.S. Blokland, R. Keppens, A\&A 532, A94 (2011)

V. Bommier, J.L. Leroy, IAU Colloq. 167: New Perspectives on Solar Prominences, vol. 150, p. 434 (1998)

S.N. Borovikov, N.V. Pogorelov, ApJ 783, L16 (2014)

D.S. Brown, R.W. Nightingale, D. Alexander et al., Sol. Phys. 216, 79 (2003)

T.-J. Cao, A.-A. Xu, C.-C. Cheng, Chin. Astron. Astrophys. 7, 1 (1983)

J. Carlyle, D.R. Williams, L. van Driel-Gesztelyi et al., ApJ 782, 87 (2014)

J. Carlyle, A. Hillier, A\&A 605, A101 (2017)

R. Casini, A. López Ariste, F. Paletou, L. Léger, ApJ 703, 114 (2009)

F. Cattaneo, D.W. Hughes, J. Fluid Mech. 196, 323 (1988)

J. Chae, S.F. Martin, H.S. Yun et al., ApJ 548, 497 (2001)

J. Chae, ApJ 714, 618 (2010)

S. Chandrasekhar, International Series of Monographs on Physics (Clarendon, Oxford, 1961)

R.A. Chevalier, ApJ 258, 790 (1982)

R.A. Chevalier, J.M. Blondin, R.T. Emmering, ApJ 392, 118 (1992)

M. Collados, A. Lagg, A. Díaz Garcí, et al., ASP Conf. Ser., vol. 368, in The Physics of Chromospheric Plasmas, ed. by P. Heinzel, I. Dorotovi, R.J. Rutten (ASP, San Francisco), p. 611 (2007)

J.L. Culhane, L.K. Harra, A.M. James et al., Sol. Phys. 243, 19 (2007)

B.J. Daly, Phys. Fluids 10, 297 (1967)

I. De Moortel, P. Browning, Philos. Trans. R. Soc. Lond. Ser. A 373, 20140269 (2015)

G. de Toma, R. Casini, J.T. Burkepile, B.C. Low, ApJ 687, L123 (2008)

A.J. Díaz, R. Soler, J.L. Ballester, ApJ 754, 41 (2012)

A.J. Díaz, E. Khomenko, M. Collados, A\&A 564, A97 (2014)

G. Dimonte, M. Schneider, Phys. Fluids 12, 304 (2000)

A.Z. Dolginov, V.M. Ostryakov, Sov. Ast. 24, 749 (1980)

J. Dudík, G. Aulanier, B. Schmieder, M. Zapiór, P. Heinzel, ApJ 761, 9 (2012)

O. Engvold, Sol. Phys. 70, 315 (1981)

M.S. Freed, D.E. McKenzie, D.W. Longcope, M. Wilburn, ApJ 818, 57 (2016)

H.R. Gilbert, A.R. Inglis, M.L. Mays et al., ApJ 776, L12 (2013)

J.P.H. Goedbloed, S. Poedts, in Principles of Magnetohydrodynamics, by J.P.H. Goedbloed, S. Poedts (Cambridge University Press, Cambridge, 2004)

J.P. Goedbloed, R. Keppens, S. Poedts, in Advanced Magnetohydrodynamics, by J.P. Goedbloed, R. Keppens, S. Poedts (Cambridge University Press, Cambridge, 2010)

S. Gosain, C. Foullon, ApJ 761, 103 (2012)

F.T. Gratton, C.J. Farrugia, S.W.H. Cowley, J. Geophys. Res. 101, 4929 (1996)

S. Gunár, P. Schwartz, J. Dudík et al., A\&A 567, A123 (2014)

S. Gunar, D.H. Mackay, ApJ 803, 64 (2015) 
L.-J. Guo, Y.-M. Huang, A. Bhattacharjee, D.E. Innes, ApJ 796, L29 (2014)

I. Hachisu, T. Matsuda, K. Nomoto, T. Shigeyama, ApJ 390, 230 (1992)

J.W. Harvey, Ph.D. Thesis (1969)

P. Heinzel, B. Schmieder, F. Fárník et al., ApJ 686, 1383-1396 (2008)

P. Heinzel, S. Gunár, U. Anzer, A\&A 579, A16 (2015)

J.J. Hester, J.M. Stone, P.A. Scowen et al., ApJ 456, 225 (1996)

A. Hillier, H. Isobe, K. Shibata, T. Berger, ApJ 736, L1 (2011)

A. Hillier, T. Berger, H. Isobe, K. Shibata, ApJ 746, 120 (2012a)

A. Hillier, H. Isobe, K. Shibata, T. Berger, ApJ 756, 110 (2012b)

A. Hillier, R. Hillier, D. Tripathi, ApJ 761, 106 (2012c)

A. Hillier, R.J. Morton, R. Erdélyi, ApJ 779, L16 (2013)

A. Hillier, A. van Ballegooijen, ApJ 766, 126 (2013)

A.S. Hillier, MNRAS 462, 2256 (2016)

A. Hillier, T. Matsumoto, K. Ichimoto, A\&A 597, A111 (2017)

N. Hurlburt, T. Berger, Hinode-3: The 3rd Hinode Science Meeting 454, 137 (2012)

D.E. Innes, R.H. Cameron, L. Fletcher, B. Inhester, S.K. Solanki, A\&A 540, L10 (2012)

D.E. Innes, L.-J. Guo, A. Bhattacharjee, Y.-M. Huang, D. Schmit, ApJ 796, 27 (2014)

D.E. Innes, P. Heinrich, B. Inhester, L.-J. Guo, A\&A 592, A17 (2016)

H. Isobe, T. Miyagoshi, K. Shibata, T. Yokoyama, Nature 434, 478 (2005)

H. Isobe, T. Miyagoshi, K. Shibata, T. Yokoyama, PASJ 58, 423 (2006)

B.-I. Jun, M.L. Norman, J.M. Stone, ApJ 453, 332 (1995)

B.-I. Jun, T.W. Jones, M.L. Norman, ApJ 468, L59 (1996)

B.-I. Jun, M.L. Norman, ApJ 465, 800 (1996)

T. Kaneko, T. Yokoyama, ApJ 806, 115 (2015a)

T. Kaneko, M. Goossens, R. Soler et al., ApJ 812, 121 (2015)

T. Kaneko, T. Yokoyama, ApJ 845, 12 (2017)

J.T. Karpen, S.K. Antiochos, ApJ 676, 658-671 (2008)

M.C. Kelley et al., Geophys. Res. Lett. 3, 448 (1976)

R. Keppens, C. Xia, O. Porth, ApJ 806, L13 (2015)

E. Khomenko, A. Díaz, A. de Vicente, M. Collados, M. Luna, A\&A 565, A45 (2014)

E. Khomenko, M. Collados, A.J. Díaz, ApJ 823, 132 (2016)

R. Kippenhahn, A. Schlüter, ZAp 43, 36 (1957)

T. Kosugi, K. Matsuzaki, T. Sakao et al., Sol. Phys. 243, 3 (2007)

M. Kruskal, M. Schwarzschild, Proc. R. Soc. Lond. Ser. A 223, 348 (1954)

A.K. Kulkarni, M.M. Romanova, MNRAS 386, 673 (2008)

M. Kuperus, M.A. Raadu, A\&A 31, 189 (1974)

N. Labrosse, P. Heinzel, J.-C. Vial et al., Space Sci. Rev. 151, 243 (2010)

N. Labrosse, B. Schmieder, P. Heinzel, T. Watanabe, A\&A 531, A69 (2011)

J.R. Lemen, A.M. Title, D.J. Akin et al., Sol. Phys. 275, 17 (2012)

S. Liberatore, S. Bouquet, Phys. Fluids 20, 116101 (2008)

S. Liberatore, S. Jaouen, E. Tabakhoff, B. Canaud, Phys. Plasmas 16, 044502 (2009)

M. Liggett, H. Zirin, Sol. Phys. 91, 259 (1984)

Y. Lin, S.F. Martin, O. Engvold, ASPC 383, 235 (2008)

E. Leonardis, S.C. Chapman, C. Foullon, ApJ 745, 185 (2012)

J.L. Leroy, Dyn. Struct. Quiescent Sol. Prominences 150, 77 (1989)

P.J. Levens, B. Schmieder, A. López Ariste et al., ApJ 826, 164 (2016)

A. López Ariste, R. Casini, A\&A 436, 325 (2005)

A. López Ariste, G. Aulanier, B. Schmieder, A. Sainz Dalda, A\&A 456, 725 (2006)

M. Luna, J.T. Karpen, C.R. DeVore, ApJ 746, 30 (2012)

M. Luna, F. Moreno-Insertis, E. Priest, ApJ 808, L23 (2015)

D.H. Mackay, J.T. Karpen, J.L. Ballester, B. Schmieder, G. Aulanier, Space Sci. Rev. 151, 333 (2010)

J. Matsumoto, Y. Masada, ApJ 772, L1 (2013)

D.E. McKenzie, ApJ 766, 39 (2013)

A. Miura, P.L. Pritchett, J. Geophys. Res. 87, 7431 (1982)

T.C. Mouschovias, ApJ 192, 37 (1974)

A.L. Moser, P.M. Bellan, Nature 482, 379 (2012)

V.M. Nakariakov, E. Verwichte, Living Rev. Sol. Phys. 2, \#3 (2005)

T.J. Okamoto, S. Tsuneta, T.E. Berger et al., Science 318, 1577 (2007) 
T.J. Okamoto, S. Tsuneta, T.E. Berger, ApJ 719, 583 (2010)

T.J. Okamoto, P. Antolin, B. De Pontieu et al., ApJ 809, 71 (2015)

T.J. Okamoto, W. Liu, S. Tsuneta, ApJ 831, 126 (2016)

R. Oliver, J.L. Ballester, Sol. Phys. 206, 45 (2002)

D. Orozco Suárez, A. Asensio Ramos, J. Trujillo Bueno, ApJ 761, L25 (2012)

D. Orozco Suárez, A.J. Díaz, A. Asensio Ramos, J. Trujillo Bueno, ApJ 785, L10 (2014)

G.J.D. Petrie, B.C. Low, ApJs 159, 288 (2005)

O. Porth, S.S. Komissarov, R. Keppens, MNRAS 443, 547 (2014)

Lord Rayleigh, Scientific Papers, vol. II (Cambridge University Press, Cambridge, 1900), p. 200

F. Reale, S. Orlando, P. Testa, E. Landi, C.J. Schrijver, ApJ 797, L5 (2014)

M.S. Ruderman, J. Terradas, J.L. Ballester, ApJ 785, 110 (2014)

M.S. Ruderman, Sol. Phys. 292, 47 (2017)

M. Ryutova, T. Berger, Z. Frank, T. Tarbell, A. Title, Sol. Phys. 267, 75 (2010)

S.L. Savage, D.E. McKenzie, K.K. Reeves, ApJ 747, L40 (2012)

M.M. Scase, K.A. Baldwin, R.J.A. Hill (2016). arXiv:1603.00675

B. Schmieder, R. Chandra, A. Berlicki, P. Mein, A\&A 514, A68 (2010)

B. Schmieder, T.A. Kucera, K. Knizhnik et al., ApJ 777, 108 (2013)

A. Secchi, Le Soleil Gauthier-Villars, Paris, vols. 1, 2 (1877)

Y. Shen, Y. Liu, Y.D. Liu et al., ApJ 814, L17 (2015)

K. Shibata, T. Tajima, R.S. Steinolfson, R. Matsumoto, ApJ 345, 584 (1989)

K. Shibata, T. Tajima, R. Matsumoto, Phys. Fluids B 2, 1989 (1990)

K. Shibata, S. Nozawa, R. Matsumoto, PASJ 44, 265 (1992)

K.A.P. Singh, V. Krishan, New A 15, 119 (2010)

H.C. Spruit, R.E. Taam, ApJ 402, 593 (1993)

H.C. Spruit, R. Stehle, J.C.B. Papaloizou, MNRAS 275, 1223 (1995)

G. Stellmacher, E. Wiehr, A\&A 24, 321 (1973)

Z. Sturrock, A.W. Hood, V. Archontis, C.M. McNeill, A\&A 582, A76 (2015)

J.M. Stone, T. Gardiner, Phys. Fluids 19, 094104 (2007)

J.M. Stone, T. Gardiner, ApJ 671, 1726 (2007)

Y. Su, A. van Ballegooijen, ApJ 757, 168 (2012)

Y. Su, P. Gömöry, A. Veronig et al., ApJ 785, L2 (2014)

T. Tajima, K. Shibata, Plasma astrophysics (Perseus, Cambridge, 2002). (Frontiers in physics, vol. 98) QB 462.7.T34

H. Takahashi et al., Ann. Geophys. 27, 1477 (2009)

T. Takahashi, A. Asai, K. Shibata, ApJ 801, 37 (2015)

S. Takasao, H. Isobe, K. Shibata, PASJ 65, 62 (2013)

E. Tandberg-Hanssen, Astrophysics and Space Science Library, 199 (Kluwer Academic, Dordrecht, 1995)

G. Taylor, R. Soc. Lond. Proc. Ser. A 201, 192 (1950)

J. Terradas, R. Oliver, J.L. Ballester, A\&A 541, A102 (2012)

J. Terradas, R. Soler, A.J. Díaz, R. Oliver, J.L. Ballester, ApJ 778, 49 (2013)

J. Terradas, R. Soler, M. Luna, R. Oliver, J.L. Ballester, ApJ 799, 94 (2015)

J. Terradas, R. Soler, M. Luna et al., ApJ 820, 125 (2016)

V.S. Titov, P. Démoulin, A\&A 351, 707 (1999)

S. Toriumi, T. Yokoyama, A\&A 539, A22 (2012)

S. Tsuneta, K. Ichimoto, Y. Katsukawa et al., Sol. Phys. 249, 167 (2008)

A.A. van Ballegooijen, S.R. Cranmer, ApJ 711, 164 (2010)

L. van Driel-Gesztelyi, D. Baker, T. Török et al., ApJ 788, 85 (2014)

M. van Dyke, NASA STI/Recon Tech. Rep. A 75, 46926 (1975)

D.R. Williams, D. Baker, L. van Driel-Gesztelyi, ApJ 764, 165 (2013)

C. Xia, R. Keppens, ApJ 823, 22 (2016a)

C. Xia, R. Keppens, ApJ 825, L29 (2016b)

T. Yokoyama, H. Shinagawa, H. Jin, J. Geophys. Res. (Space Phys.) 119, 10 (2014)

D.L. Youngs, Phys. D Nonlinear Phenom. 37, 270 (1989)

X. Zhai, P.M. Bellan, Phys. Plasmas 23, 032121 (2016) 